\title{
The Difference in Narrow Fe K $\alpha$ Line Emission Between Seyfert 1 and Seyfert 2 Galaxies
}

\author{
Teng Liu \& Jun-Xian Wang \\ CAS Key Laboratory for Research in Galaxies and Cosmology, Department of Astronomy, \\ University of Science and Technology of China; lewtonstein@gmail.com; jxw@ustc.edu.cn
}

\begin{abstract}
We compile a sample of 89 Seyfert galaxies with both [OIV] $25.89 \mu \mathrm{m}$ line luminosities observed by Spitzer IRS and X-ray spectra observed by XMM-Newton EPIC. Using [OIV] emission as proxy of AGN intrinsic luminosity, we find that although type 2 AGNs have higher line equivalent width, the narrow Fe K $\alpha$ line in Compton-Thin and Compton-Thick Seyfert 2 galaxies are $2.9_{-0.6}^{+0.8}$ and $5.6_{-1.4}^{+1.9}$ times weaker in terms of luminosity than Seyfert 1 galaxies respectively. This indicates different correction factors need to be applied for various types of AGNs before the narrow $\mathrm{Fe} \mathrm{K} \alpha$ line luminosity could serve as intrinsic AGN luminosity indicator. We also find Seyfert 1 galaxies in our sample have on average marginally larger line width and higher line centroid energy, suggesting contamination from highly ionized Fe line or broader line emission from much smaller radius, but this effect is too weak to explain the large difference in narrow Fe K $\alpha$ line luminosity between type 1 and type 2 AGNs. This is the first observational evidence showing the narrow $\mathrm{Fe} \mathrm{K} \alpha$ line emission in AGNs is anisotropic. The observed difference is consistent with theoretical calculations assuming a smoothly distributed obscuring torus, and could provide independent constraints on the clumpiness of the torus.
\end{abstract}

Subject headings: galaxies: active - galaxies: nuclei - galaxies: Seyfert - X-rays: galaxies - line: profiles

\section{Introduction}

Narrow Fe K $\alpha$ emission line cores are common in the X-ray spectra of both type 1 and type 2 Active Galactic Nuclei (AGNs) (e.g. see Bianchi et al. 2009; LaMassa et al. 2009 for most recent studies). Such narrow lines have been traditionally associated with an origin in distant matter, especially the putative obscuring torus. Comprehensive and systematic studies of the narrow Fe $\mathrm{K} \alpha$ line in Seyfert 1 galaxies with the Chandra High Energy Grating (HEG) were presented by Yaqoob \& Padmanabhan (2004) and Shu et al. (2010). Shu et al. reported a mean line width of $2060 \mathrm{~km} / \mathrm{s}$, supporting an origin in distant matter. Meanwhile, considering the observed larger scatter in line width from source to source, contributions from inner region (such as the outer part 
of the accretion disk) could not be ruled out. Although the velocity widths in some of the sources were found consistent with those of the optical broad emission lines (also see Yaqoob et al. 2001; Bianchi et al. 2003), Nandra (2006) found no correlation between the Fe K $\alpha$ core width and the BLR (specifically $\mathrm{H} \beta$ ) line width, eliminating the BLR as a general origin of the Fe K $\alpha$ core (also see Shu et al. 2010). The lack of correlation between the equivalent width of narrow Fe K $\alpha$ emission and $\mathrm{EW}(\mathrm{CIV})$ (Wu et al. 2009) gives weight to this scenario.

Type 2 AGNs are believed viewed at inclination larger than type 1 AGN in the framework of unification model (e.g., Antonucci 1993). In such model, the line of sight of the observer to type 2 AGNs is obscured by an equatorial torus, which blocks the central accretion disk and the broad emission line region. The column density of the obscuration in type 2 AGNs spans from $N_{H} \sim 10^{22} \mathrm{~cm}^{-2}$ to $N_{H}>10^{24} \mathrm{~cm}^{-2}$ (Compton-Thick) (Bassani et al. 1999). The narrow Fe K $\alpha$ emission lines often show higher equivalent width (EW) in type 2 AGNs, presumably due to the attenuation of the underlying continuum (e.g. LaMassa et al. 2009). Particularly, in type 2 AGNs with extreme X-ray obscuration (i.e. Compton-Thick), the narrow Fe K $\alpha$ lines even appear as the most prominent feature in the hard X-ray spectra and could have EW as large as several keV (e.g. Levenson et al. 2006). As the narrow Fe $\mathrm{K} \alpha$ emission likely arises from the putative torus rather than the BLR, studies of the narrow core could place constraints on the unified model.

Although the luminosity of the Fe $\mathrm{K} \alpha$ line has been suggested to be an indicator of intrinsic AGN flux (Ptak et al. 2003; Levenson et al. 2006; LaMassa et al. 2009), it is still unclear whether the narrow $\mathrm{Fe} \mathrm{K} \alpha$ line emission itself (i.e. the line flux but not line $\mathrm{EW}$ ) depends on inclination. To answer this question, we present in this paper a first systematic comparison of the narrow Fe K $\alpha$ lines in type 1 and type 2 AGNs. To perform such comparison, an intrinsic luminosity indicator of the central engine is required. Hard X-ray luminosity or reddening-corrected $[\mathrm{O}$ III] were commonly used as isotropic luminosity indicators in various studies (e.g. Bassani et al. 1999; Diamond-Stanic et al. 2009; Meléndez et al. 2008b). However, in the case of Compton-Thick sources which we are interested in particularly, hard X-ray luminosity is also strongly attenuated due to Compton scattering. The dust reddening to [O III] brings extra uncertainty to the measurement of intrinsic [O III] luminosity. Furthermore, the [OIII] emission could be partially obscured in type 2 AGNs (Zhang et al. 2008), making reddening correction procedure more difficult. In this paper, we select to use [OIV] $25.89 \mu \mathrm{m}$ line as an intrinsic luminosity indicator. Since [OIV] $25.89 \mu \mathrm{m}$ has relatively higher ionization potential $(54.9 \mathrm{eV})$, it's less affected by star formation. It is also significantly less affected by extinction than [OIII] $\left(A_{v} \sim 3-9\right.$ corresponds to $A_{25.89 \mu m} \sim 0.06-0.18$; Goulding \& Alexander 2009). Meléndez et al. (2008b) have found that both [O IV] and [O III] luminosity correlate well with the very hard X-ray luminosity (14-195 keV) as measured by the SWIFT/BAT. Meanwhile Diamond-Stanic et al. (2009) reported that the [OIV] luminosity distributions are indistinguishable for obscured and unobscured Seyferts, while [OIII] luminosities are systematically smaller for obscured Seyferts. These confirm that [OIV] could serve as a reddening-free isotropic luminosity indicator. Also, $[\mathrm{OIV}]$ represents an improvement over the use of infrared continuum given the difficulty in isolating the AGN continuum from the host galaxy 
emission (Lutz et al. 2004).

\section{Sample and Data Reduction}

\subsection{Sample Selection}

[OIV] $25.89 \mu \mathrm{m}$ emission lines have been detected in large number of AGNs by the Infrared Spectrograph (IRS) on board the Spitzer Space Telescope in the first Long-Low (LL1, $19.5 \sim 38.0 \mu m$ ) IRS order. Diamond-Stanic et al. (2009) and Meléndez et al. (2008a) presented two large AGN samples (respectively 89 and 103 Seyferts) with [OIV] measurements. We crosscorrelated the two samples with 2 XMM catalogue (the second comprehensive catalogue of serendipitous X-ray sources from XMM-Newton), which was released on 2007 August 22nd (Watson et al. 2009). By excluding sources with less than 200 total EPIC counts in $2-12 \mathrm{keV}$, our final sample consists of 182 XMM observations of 89 Seyferts. We note that the final sample is never a homogeneous one, since it relies on the availability of both archival Spitzer spectral data and XMM data of individual sources. However, most (if not all) of the Spitzer and XMM observations were planned independent of their OIV emission and Fe $\mathrm{K} \alpha$ line emission. Our study, which is focus on $\mathrm{OIV}$ and $\mathrm{Fe} \mathrm{K} \alpha$ line emission, is thus free from the diverse selection effects. The cutoff in $\mathrm{X}$-ray counts (> 200 counts, 2-12 keV) excludes weaker X-ray sources ( compared with OIV emission). However, this effect is also independent of $\mathrm{Fe} \mathrm{K} \alpha$ line, since even for Compton-thick sources with $\mathrm{Fe}$ $\mathrm{K} \alpha \mathrm{EW}$ of $1 \mathrm{keV}$, the emission line could only contribute $13 \%$ to $2-12 \mathrm{keV}$ EPIC counts (assuming a pure reflection model pexrav with a photon index of 2 and a folded energy of $100 \mathrm{keV}$, plus a narrow gauss line at $6.4 \mathrm{keV}$ whose $\mathrm{EW}$ is set to be $1 \mathrm{keV}$ in xspec ).

We gathered the redshifts and coordinates for each source from Simbad. X-ray classification of type 2 AGNs (to distinguish between Compton-thin and Compton-thick) requires not only proper spectral fitting to full band X-ray spectra, but also much more complex models than the ones adopted in this paper, including partial covering absorber, soft X-ray excess, scattering component, contamination from host galaxy, etc. Furthermore, low quality spectra could make the situation more difficult, and in many cases one need additional diagnostics, such as Fe K $\alpha$ line EW and $\mathrm{T}$ ratio $\left(F_{2-10 \mathrm{keV}} / F_{[O I I I]}\right.$, see Bassani et al. 1999). Thus a uniform summary of X-ray classifications is unavailable. Fortunately detailed X-ray studies for all but one Seyfert 2 galaxies in our sample have been published, and in this paper we choose to quote their X-ray classifications from the literature.

The X-ray absorption column density of Seyfert 2 galaxy NGC 777 was not available from literature, and we classified it as Compton-Thin based on spectral fitting to XMM data. We divided the sample into 3 subsamples: Seyfert 1s (including Sy 1.2-1.5s, 33 sources), Compton-Thin Seyfert 2s (including Sy1.8-1.9s, 35 sources) and Compton-Thick Seyfert $2 \mathrm{~s}$ (with $N_{H}>10^{24} \mathrm{~cm}^{-2}$, 21 sources). In panel A of Fig. 1 we plot the histogram distribution of [OIV] luminosity for three 
subsamples. Excluding 6 upper limits of $L_{[O I V]}$, we processed KS test (short for KolmogorovSmirnov test, which compares two samples under the null hypothesis that the samples are drawn from the same distribution 1 ) on [OIV] luminosity between subsamples. No significant difference was found (the differences between Sy1s and Compton-Thin Sy2s, Sy1s and Compton-Thick Sy2s, Compton-Thin Sy2s and Compton-Thick Sy2s are at confidence levels of respectively 89.2\%, $14.6 \%, 85.5 \%$, with corresponding D values of $0.29,0.17,0.32)$, ensuring we are comparing subsamples in same intrinsic luminosity range. Throughout this paper, we adopt cosmological parameters $H_{0}=70 \mathrm{kms}^{-1} \mathrm{Mpc}^{-1}, \Omega_{m}=0.27$, and $\Omega_{\lambda}=0.73$. The sample is given in Table 1 .

\subsection{Data Reduction}

We used XMM-SAS (Science Analysis Software) version 9.0.0 and calibration files as of 2009 August to reduce archival data. Each observation was processed using the pipeline "epchain" and "emchain". All the events were filtered to include only those with XMM-SAS quality flag $\mathrm{FLAG}==0$ (\#XMMEA_EM) for PN ( MOS1 and MOS2), and cleaned from flaring background with the task "espfilt". The task "espfilt" failed in 1 out of 9 exposures of Mrk 3, and this exposure (0009220301PNU002) was excluded from our further analysis. Another 4 observations of 3C 273 in PrimeFull mode were eliminated due to serious pileup. Generally we extracted event files comprising single- and double-pixel events (PATTERN $\leq 4)$ for PN, and single- to quadruple-pixel events (PATTERN $\leq 12$ ) for MOS1 and MOS2. However, in 90 exposures (Table 1), moderate pileup was identified using the task "epatplot", therefore only single-pixel events were extracted for these 90 exposures to reduce the pileup effect (Ballet 1999).

The level of pileup was also estimated through PIMMS2 2 using observed spectra, and we found a maximum pileup fraction of $4 \%$ in PN detectors in our sample. Although such level of pileup could still alter observed spectral shape, the effect on the fitting to narrow Fe K $\alpha$ line, which is the aim of this work, is negligible (also see $§ 2.3$ ).

We defined the source region as a circle with radius of $40^{\prime \prime}$ centered at the source position, and the background regions as three circles with the same radii around the source region, being central symmetric as much as possible, and kept away from the CCD edges, the out-of-time events strips and other sources (as illustrated in Fig. 2). When we were unable to avoid these disruptive features, the radii of the source or background regions were reduced down to $25^{\prime \prime}$ (see Table1). We generated the source and background spectra together with the appropriate redistribution matrix and ancillary response file from the source and background regions for each exposure, using the task "especget". Then for each source, to make the Fe K $\alpha$ feature prominent as it may not appear in

\footnotetext{
${ }^{1}$ A statistically significant difference corresponds to a confidence level of $95 \%$ or higher

${ }^{2}$ http://heasarc.nasa.gov/docs/software/tools/pimms.html
} 
individual observations, the PN spectra and the MOS1, MOS2 spectra from different observations were respectively combined, using the Ftools task "addspec", (see Section 3.2 for further discussion on the combination). The combined MOS1 and MOS2 spectra will be referred to as MOS hereafter.

\section{3. $\quad$ Spectral Fitting}

All spectra were grouped so that each channel contains at least one count, and spectral fitting were performed with C-statistic using Xspec version 12.4.0. As our major goal in this work is to measure the flux of the narrow $\mathrm{Fe} \mathrm{K} \alpha$ line, we fitted the $5-10 \mathrm{keV}$ spectra with a simple model uniformly. A Gaussian line was used to fit the narrow Fe $\mathrm{K} \alpha$ line, and the continuum were fitted with an absorbed powerlaw (zwabs* powerlaw+zgauss). Some examples of our spectral fitting are given in Fig. 3 ,

Whenever available, PN and MOS spectra were fitted simultaneously with same model parameters, except for the powerlaw and Gaussian line normalizations, which were set free to account for the discrepancy among detector calibrations. Normalizations from PN spectra are presented in these cases. Pileup effects would be more significant in MOS detectors, which could alter the continuum spectral shape. For these MOS data suffering obvious pileup (see §2.2), continuum parameters (photon index and absorption) were also setting free.

In Table 1 we list the best-fit parameter for the narrow Fe K $\alpha$ lines. For 25 of the sources, the detection of narrow Fe K $\alpha$ lines were insignificant (with F-statistic confidence level < 99\%). In NGC7314 and I ZW 1, the centroid energies (rest-frame) of the detected gauss line are larger than $6.4 \mathrm{keV}$ at $>3 \sigma$ leve 3 , which seem to be due to ionized Fe lines, and extra gauss line at $\sim 6.4 \mathrm{keV}$ is statistically not required. For these 27 sources where the detection of the Fe K $\alpha$ line was not significant, we provide upper limit to their narrow Fe $\mathrm{K} \alpha$ line flux by fitting with a gauss line with central energy fixed at $6.4 \mathrm{keV}$ (rest-frame) and the $\sigma$ at $44 \mathrm{eV}$ (the median $\sigma$ of the detected lines for the full sample, see panel B of Fig. 1). Fixing the energy and $\sigma$ to the median values found for each source's corresponding sub-population does not alter our results in this paper. In several sources, extra gaussian lines were statistically required (with F-statistic confidence $>99 \%$ ), most of these lines could be attributed to either broader Fe K $\alpha$ line, ionized Fe K $\alpha$ line or Fe K $\beta$ line (see Table 2).

The spectral fitting to the narrow Fe K $\alpha$ line could be significantly affected by continuum complexities. To quantify this effect, we generated 390 artificial spectra based on various continuum models plus narrow lines, and apply our uniform model (wabs*powerlaw+zgauss) to fit the artificial spectra between 5 and $10 \mathrm{keV}$. The models we adopted for simulations include A) partially absorbed powerlaw (pcfabs* powerlaw+zgauss model in Xspec, with covering factor varying from 0 to 1 , and $n_{H}$ between $10^{21} \sim 10^{24} \mathrm{~cm}^{-2}$ ), and B) absorbed powerlaw plus reflection component

\footnotetext{
${ }^{3} 3 \sigma$ errors of centroid energies were calculated through $x$ spec, but only $90 \%$ errors were presented in the tables.
} 
$\left(\right.$ wabs ${ }^{*}$ powerlaw + pexrav + zgauss in Xspec, with $n_{H}$ varying between $10^{21} \sim 10^{24} \mathrm{~cm}^{-2}$, and relative strength of the direct component to the reflected component varying from 0 to 10). The line width and $\mathrm{EW}$ of the simulated narrow $\mathrm{Fe} \mathrm{K} \alpha$ line were selected to cover the whole range of the final best-fit parameters in our sample (see Table1). Through the simulations we find that by restricting the fitting to $5-10 \mathrm{keV}$ range, we can recover the simulated narrow $\mathrm{Fe} \mathrm{K} \alpha$ lines with systematical deviation $<2 \%$. Adopting wider spectral range, such as $2-10 \mathrm{keV}$, would yield significant bias to the narrow $\mathrm{Fe} \mathrm{K} \alpha$ line measurement (as large as 300\%), due to the improper fitting to the underlying continuum.

\section{Statistical Distributions}

\subsection{Centroid Energy, Line Width and Line Equivalent Width}

In panel $\mathrm{C}$ of Fig. 1 we first plot the centroid energy distribution for narrow $\mathrm{Fe} \mathrm{K} \alpha$ lines in three subsamples. We find while the line centroid energies in type 2 AGNs are generally consistent with $6.4 \mathrm{keV}$, those in type 1 AGNs tend to be slightly higher. KS test indicates that the difference in the narrow $\mathrm{Fe} \mathrm{K} \alpha$ line central energy distributions between type 1 AGNs and type 2 AGNs is statistically marginal (with a confidence level of $98.4 \%$ and $\mathrm{D}=0.385$ ). We also derived a weighted mean centroid energy of $6.409 \pm 0.002 \mathrm{keV}$ for type 1 AGNs, and $6.402 \pm 0.004,6.401 \pm 0.002 \mathrm{keV}$ for Compton-Thin and Compton-Thick Sy2s respectively. Higher centroid energies of the detected Fe $\mathrm{K} \alpha$ lines of Sy1s listed in Table 1 could be due to contamination from unresolved highly ionized Fe line or inner region (such as the accretion disk) component which is Doppler broadened and affected due to general relativity (Fabian et al. 1989)4, and such components could be weaker in type 2 AGNs due to strong obscuration from the torus.

We note that by studying large samples of type 1 AGNs with HETG observations, Yaqoob \& Padmanabhan (2004) and Shu et al. (2010) have demonstrated that the centroid Fe K $\alpha$ line energies in type 1 AGN strongly peak at $6.4 \mathrm{keV}$. This indicates that the contamination to the narrow Fe K $\alpha$ line core is much weaker in HETG spectra due to its much better spectral resolution.

We also compared the Fe $\mathrm{K} \alpha$ line widths between type 1 and type 2 sources. Due to the limited spectral resolution of XMM PN/MOS, many of the narrow lines are unresolved, and only upper limits were given (see Table 1). The line width distributions are plotted in panel B of Fig. 1, where can see slightly larger line width in type 1 AGNs compared with type 2 sources. Making use of ASURV (The Astronomy Survival Analysis; Feigelson \& Nelson 1985), which can be used in the presence of upper limit data, and will be used in this paper whenever upper limits exist in the data, we performed Peto-Prentice Generalized Wilcoxon test, and found that the line width distribution in type 1 AGNs differs from that of type 2 AGNs with a confidence level of $92 \%$. This pattern is

\footnotetext{
${ }^{4}$ Modeling the broad component is beyond the scope of this paper. Systematic studies of broad Fe K $\alpha$ line emission from accretion disk are available in Brenneman \& Reynolds (2009) and Nandra et al. (2007).
} 
consistent with the centroid energy distribution, suggesting the narrow Fe K $\alpha$ line in XMM spectra of type 1 AGNs is likely polluted by either highly ionized Fe line or broader emission from smaller radius.

Consistent with previous studies (e.g. Bassani et al. 1999), we do find larger Fe K $\alpha$ line equivalent width in type 2 AGNs (see panel D of Fig. 1). Through Peto-Prentice Generalized Wilcoxon test (ASURV), we find the Fe K $\alpha$ line equivalent width distribution in type 1 AGNs differs from that of Compton-thin and Compton-thick Sy2s with confidence level of $70 \%$ and $>99.99 \%$ respectively.

To examine the quoted X-ray classification, we reproduced the anti-correlation between the Fe $\mathrm{K} \alpha$ equivalent width and the $2-10 \mathrm{keV}$ luminosity normalized to the intrinsic AGN luminosity found by Bassani et al. (1999), replacing reddening corrected [OIII] luminosity with [OIV] luminosity as proxy of the intrinsic luminosity (see Fig. 4). The $2-10 \mathrm{keV}$ luminosities were calculated by fitting the 2-10 keV band spectra with two independent absorbed powerlaw and a gaussian line. We note such fitting could be unphysical to many sources, and is only valid to estimate the observed $2-10 \mathrm{keV}$ fluxes. In Fig. 4, the concentration of Compton-Thick sources at the upper left corner confirmed their classifications, including three Compton-Thick sources (NGC3982, NGC4501, NGC7674) with Fe K $\alpha$ line non-detected (upper limits to Fe K $\alpha$ line EW were plotted).

Bassani et al. (1999) adopted a threshold of $\mathrm{F}_{2-10 \mathrm{keV}} / \mathrm{F}_{[O I I I], c o r r}<1$ to identify Comptonthick sources. LaMassa et al. (2010) reported an average ratio of $\log \left(\mathrm{F}_{[O I V]} / \mathrm{F}_{[O I I I], \text { corr }}\right)=-0.82$ for $12 \mu \mathrm{m}$ selected Sy2s. Thus $\log L_{2-10 \mathrm{keV}} / L_{[O I V]}<0.82$ can also be used as a boundary for ComptonThick sources in our sample. In Fig. 4 we plot $\log L_{2-10 k e V} / L_{[O I V]}=1$ and $\log E W_{F e}=2.5$ (which include all Compton-thick sources) to delineate the Compton-thin/Compton-thick boundaries. Note there are four Compton-Thin sources fall in the Compton-Thick region. They turn out to be "nearly" Compton-Thick with $\log n_{H}>23$ (NGC1358: 23.4; NGC7479: 23.6; MCG-2-8-39: 23.7; MRK273: 23.8. See references in Table. 1). We conclude that the quoted X-ray classifications are generally consistent with the measured Fe K $\alpha$ line EW and $\log L_{2-10 k e V} / L_{[O I V]}$. Re-classifying Seyfert 2 galaxies based on Fig. 4 will not alter the results presented in this paper.

\subsection{Line Luminosity}

In the upper panel of Fig. 5 we plot the narrow $\mathrm{Fe} \mathrm{K} \alpha$ line luminosity versus [OIV] line luminosity for our samples. We first perform Buckley-James linear regression on the $\log L_{F e}-$ $\log L_{[O I V]}$ distribution for each subsample with ASURV. Six sources with only $L_{[O I V]}$ upper limit were shown in Fig. 5, but excluded from statistical analysis since ASURV can not handle upper limits in both data sets. The best-fit slopes are:

$$
\begin{array}{ll}
\text { Sy1-1.5: } & \log L_{F e}=\log L_{[O I V]} \times(0.89 \pm 0.09)+4.62 \pm 0.40 \\
\text { C-Thin Sy2: } & \log L_{F e}=\log L_{[O I V]} \times(0.92 \pm 0.08)+3.02 \pm 0.36 \\
\text { C-Thick Sy2: } & \log L_{F e}=\log L_{[O I V]} \times(0.73 \pm 0.12)+10.24 \pm 0.45
\end{array}
$$


We note that excluding the extremely faint Seyfert 1 M81 does not significantly alter the best-fit slope and other results presented in this paper.

To explore the difference in $L_{[\mathrm{Fe}]}$ between subsamples, we compare the residuals of $\log L_{F e}$ from the best-fit line of $\log L_{F e}-\log L_{[O I V]}$ for Sy1s for each subsample. This is valid since the three subsamples show consistent $L_{[O I V]}$ distributions (see $\S 2.1$ and Fig. 66). With PetoPrentice Generalized Wilcoxon Test (ASURV), we found significant differences between Sy1s and Compton-thin Sy2s, and between Sy1s and Compton-thick Sy2s in the $\log L_{F e}$ residuals (with confidence level of $99.98 \%$ and $>99.99 \%$ respectively, see Fig. 6). This implies the distribution of $\log L_{F e}-\log L_{[O I V]}$ also differs statistically significantly between Sy1s and Sy2s. The difference in $\log L_{F e}$ residuals between Compton-Thin and Compton-Thick Sy2s is only marginal (with a significance level of $86 \%$ ). By comparing the mean values of the $\log L_{F e}$ residuals in subsamples we find the narrow $\mathrm{Fe} \mathrm{K} \alpha$ lines in Sy1s are $2.9_{-0.6}^{+0.8}$ times stronger in terms of luminosity than that of Compton-Thin Sy2s, and 5.6 $6_{-1.4}^{+1.9}$ times stronger than that of Compton-Thick Sy2s. Simply comparing $\log L_{\mathrm{Fe}} / \log L_{[O I V]}$ between subsamples yields consistent results, that the narrow Fe $\mathrm{K} \alpha$ lines in Sy1s are 2.6 $6_{-0.6}^{+0.8}$ times stronger than that of Compton-Thin Sy2s, and 5.7 $7_{-1.6}^{+2.1}$ times stronger than that of Compton-Thick Sy2s.

For comparison, we plot $6.4 \mathrm{keV}$ monochromatic luminosity $\left(L_{F e} / E W_{F e}\right)$ versus $L_{[O I V]}$ for three subsamples in the lower panel of Fig. 5. Adopting the same methods used above, we find that the best fitting slopes for Sy1s, Compton-Thin Sy2s and Compton-Thick Sy2s are respectively $1.03 \pm 0.07,0.90 \pm 0.10$ and $0.89 \pm 0.11$, and the $6.4 \mathrm{keV}$ monochromatic luminosity in Sy1s are $4.2_{-1.2}^{+1.6}$ times stronger than that of Compton-Thin Sy2s, and 43.2 $2_{-11.7}^{+16.0}$ times that of Compton-Thick Sy2s. These decrements, if simply attributed to photo-electric absorption, require column densities of $8.6_{-2.1}^{+1.9} \times 10^{23} \mathrm{~cm}^{-2}$ and $2.3_{-0.2}^{+0.2} \times 10^{24} \mathrm{~cm}^{-2}$ respectively (calculated using a simple photo-electric absorbing model wabs*gauss in Xspec, with the line energy and $\sigma$ fixed at $6.4 \mathrm{keV}$ and $0 \mathrm{eV}$ ).

To examine whether our fitting could be biased by spectral combination which could produce spurious results for variable sources, we repeat our spectral fit to individual observations. Discarding the observations in which narrow $\mathrm{Fe} \mathrm{K} \alpha$ line is no longer detectable, we calculated the weighted mean line flux for each source. Fig. 7 compares the weighted mean line flux from individual observations with the line flux from the combined spectra, between which no obvious difference is found.

\section{Discussion}

By normalizing to [OIV], which serves as an isotropic indicator of AGN luminosity, we find that the narrow $\mathrm{Fe} \mathrm{K} \alpha$ line in Compton-Thin and Compton-Thick Sy2s are $2.9_{-0.6}^{+0.8}$ and $5.6_{-1.4}^{+1.9}$ 
times weaker than Sy1s respectively. We note that based on the X-ray reprocessing torus model of Krolik et al. (1994), Levenson et al. (2006) performed numerical simulations to 7 Compton-Thick AGNs to estimate their intrinsic 2 - $10 \mathrm{keV}$ luminosity from observed Fe K $\alpha$ EW and luminosity. In their model, the obscuring torus is cylindrical symmetric, filled with constant density material, and has a square or rectangular cross-section allowing for unobstructed views over the half-opening angle. They obtained a typical value of $\left.L_{F e} / L_{\text {intrinsic }(2-10 k e V}\right)=2 \times 10^{-3}$ for the 7 ComptonThick AGNs. Such ratio is approximately 5 times smaller than the median value of our Sy1s with Fe $\mathrm{K} \alpha$ line detected (a median line $\mathrm{EW}$ of $96 \mathrm{eV}$ could be converted to $F_{\mathrm{Fe}} / F_{2-10 \mathrm{keV}}=9.7 \times 10^{-3}$ assuming a powerlaw with photon index $\Gamma=1.9)$. Taking our observed $\left.L_{(} 2-10 \mathrm{keV}\right)$ for Sy1s presented in Table 1, we obtained a similar $F_{F e} / F_{2-10 k e V}=11.2 \times 10^{-3}, 5.6$ times larger than those for Compton-thick Sy2s presented by Levenson et al. These are remarkably consistent with our results that the observed Fe K $\alpha$ emission in Compton-Thick AGNs is 5.6 $6_{-1.4}^{+1.9}$ times weaker than type 1 AGNs.

Meanwhile we also find marginally higher centroid energy and larger line width in type 1 AGNs compared with type 2 AGNs. This indicates the narrow Fe K $\alpha$ line in XMM spectra of type 1 AGNs is likely polluted by highly ionized Fe line or broader component from smaller radius in type 1 AGNs, and such contamination is weaker in type 2 AGNs due to the torus obscuration. If highly ionized Fe line or broader component from smaller radius was responsible for the disparity in Fe K $\alpha$ luminosity between Sy1s and Sy2s, the Sy1s with larger line widths and/or higher centroid energy should have systematically higher $L_{F e} / L_{[O I V]}$ ratios. In Fig. 6 we see that Sy1 galaxies with higher Fe K $\alpha$ line centroid energy or larger line width ( compared with the median values of the Sy1s with Fe K $\alpha$ line detected) do not have obviously larger $L_{F e} / L_{[O I V]}$. KS tests show that the differences in $\log L_{F e}$ residuals in Fig. 6 between Sy1s with higher and lower centroid energy, and between Sy1s with larger and smaller line widths, are at confidence levels of $51 \%(\mathrm{D}=0.31)$ and $91 \%(\mathrm{D}=0.46)$ respectively, neither of which are statistically significant. Similar confidence levels of $51 \%(\mathrm{D}=0.31)$ and $77 \%(\mathrm{D}=0.38)$ were obtained if we simply compare $\log \left(L_{F e} / L_{[O I V]}\right)$ of Sy1 subsamples. The average $\log \left(L_{F e} / L_{[O I V]}\right)$ is $0.18 \pm 0.14$ for Syls with higher centroid energy, $-0.02 \pm 0.09$ for lower centroid energy, $0.16 \pm 0.11$ for Sy1s with larger line width, and $0.00 \pm 0.12$ for smaller line width. This indicates that the contribution from either highly ionized Fe line or broader emission from much smaller radius is too small to explain the difference we found between type 1 and type 2 AGNs.

We note that among the 26 Sy1s with narrow Fe $\mathrm{K} \alpha$ line detected in table 1, eight have both higher line centroid energy $\left(\mathrm{E}_{c}\right)$ and larger line width $(\sigma)$, and eight show lower $\mathrm{E}_{c}$ and smaller $\sigma$ ( compared with the median values of Sy1 sample). Five Sy1s show higher $\mathrm{E}_{c}$ but smaller $\sigma$, and the rest five have lower $\mathrm{E}_{c}$ but larger $\sigma$. Thus no significant correlation is found between $\mathrm{E}_{c}$ and line width. Meanwhile, contamination from unresolved ionized Fe $\mathrm{K} \alpha$ emission could produce lines with higher $\mathrm{E}_{c}$ and larger $\sigma$, those lines with smaller $\mathrm{E}_{c}$ and larger $\sigma$ are more likely due to Doppler broadening.

The Fe K $\alpha$ line emission could be determined by many factors, including the geometry, column 
density, covering factor of the line-emitting gas, element abundances, and the orientation of the observer's line of sight relative to the line-emitting structure. In the standard unified model, the only difference between type 1 and type 2 AGNs is the orientation, thus is the only plausible factor to explain the different $\mathrm{Fe} \mathrm{K} \alpha$ line emission. Observational selection effects could possibly favor smaller column density and covering factor of the torus in type 1 sources, but both effects predict weaker $\mathrm{Fe} \mathrm{K} \alpha$ emission (contrarily, larger covering factor and column density of the torus could generally enhance the narrow Fe $\mathrm{K} \alpha$ line emission in type 1 sources). Meanwhile, we do not expect significantly higher Fe abundance in type 1 AGNs, not to mention that the Fe K $\alpha$ line emission is insensitive to Fe abundance unless in optically-thin limit $\left(\mathrm{N}_{H}<10^{23} \mathrm{~cm}^{-2}\right.$, see Yaqoob et al. 2010).

Yaqoob et al. (2010) presented Monte-Carlo simulations on the production of Fe K $\alpha$ emission in toroidal neutral X-ray reprocessor (also see Murphy \& Yaqoob 2009). They clearly demonstrate that the strength of the line emission from a toroidal torus could dramatically depend on the viewing angle, as long as the column density of the reprocessor is significantly larger than $10^{23} \mathrm{~cm}^{-2}$. For instance, for column density of $10^{24}-10^{25} \mathrm{~cm}^{-2}$, Fe K $\alpha$ emission at face-on inclination could be around 3-30 times stronger than edge-on inclination (see Fig. 2 of Yaqoob et al. 2010). This is mainly because of the larger optical depth to Fe K $\alpha$ emission due to photo-electric absorption and Compton scattering at edge-on inclination. This scheme could easily produce the difference in the observed Fe K $\alpha$ line emission between type 1 and type 2 AGNs. Clearly, this scheme requires the existence of Compton-thick torus in both type 1 and type 2 AGNs, although the line of sight is free of Compton-thick obscuration in type 1 and Compton-thin type 2 AGNs.

However, if the line-emitting gas is clumpy instead of smoothly distributed, the inclination dependency of the line emission could be reduced or even smeared out (also see the Nandra \& George 1994 and Miller et al. 2009), dependent on the filling factor and covering factor of the clumpy blobs. Monte-Carlo simulations of the dust emission and radiative transfer in the clumpy torus have been performed to model infrared emission in various types of AGNs, which could produce strong constraints on the clumpiness of the dusty torus (e.g. Nenkova et al. 2008). Our results could thus provide independent constraints to the distribution of the cold gas (which produces Fe $\mathrm{K} \alpha$ line emission, and its distribution may differ from that of dust) in a clumpy torus model once detailed Monte-Carlo simulation of Fe $\mathrm{K} \alpha$ line production in such model would be available.

Our results indicate that the narrow $\mathrm{Fe} \mathrm{K} \alpha$ emission in AGNs is anisotropic, with weaker emission (although higher EW) in obscured AGNs. Extra correction factors should be applied if one uses observed Fe K $\alpha$ emission to estimate AGN's intrinsic luminosity.

The work was supported by Chinese NSF through NSFC10773010/10825312, and the Knowledge Innovation Program of CAS (Grant No. KJCX2-YW-T05). 


\section{REFERENCES}

Akylas, A., \& Georgantopoulos, I. 2009, A\&A, 500, 999

Antonucci, R. 1993, ARA\&A, 31, 473

Awaki, H., Terashima, Y., Higaki, Y., \& Fukazawa, Y. 2008, ArXiv e-prints

Balestra, I., Boller, T., Gallo, L., Lutz, D., \& Hess, S. 2005, A\&A, 442, 469

Ballantyne, D. R., Fabian, A. C., \& Iwasawa, K. 2004, MNRAS, 354, 839

Ballet, J. 1999, A\&AS, 135, 371

Bassani, L., Dadina, M., Maiolino, R., Salvati, M., Risaliti, G., della Ceca, R., Matt, G., \& Zamorani, G. 1999, ApJS, 121, 473

Beckmann, V., Gehrels, N., Favre, P., Walter, R., Courvoisier, T., Petrucci, P., \& Malzac, J. 2004, ApJ, 614, 641

Bianchi, S., Guainazzi, M., Matt, G., Fonseca Bonilla, N., \& Ponti, G. 2009, A\&A, 495, 421

Bianchi, S., Matt, G., Balestra, I., \& Perola, G. C. 2003, A\&A, 407, L21

Braito, V., et al. 2004, A\&A, 420, 79

Branduardi-Raymont, G., Blustin, A. J., Wu, K., Kaastra, J. S., Kahn, S. M., \& Deluit, S. 2002, in High Resolution X-ray Spectroscopy with XMM-Newton and Chandra, ed. G. BranduardiRaymont

Brenneman, L. W., \& Reynolds, C. S. 2009, ApJ, 702, 1367

Brightman, M., \& Nandra, K. 2008, MNRAS, 390, 1241

Cardamone, C. N., Moran, E. C., \& Kay, L. E. 2007, AJ, 134, 1263

Churazov, E., Forman, W., Jones, C., \& Böhringer, H. 2003, ApJ, 590, 225

Dewangan, G. C., Griffiths, R. E., Di Matteo, T., \& Schurch, N. J. 2004, ApJ, 607, 788

Diamond-Stanic, A. M., Rieke, G. H., \& Rigby, J. R. 2009, ApJ, 698, 623

Fabian, A. C., Rees, M. J., Stella, L., \& White, N. E. 1989, MNRAS, 238, 729

Fabian, A. C., \& Vaughan, S. 2003, MNRAS, 340, L28

Feigelson, E. D., \& Nelson, P. I. 1985, ApJ, 293, 192

Fukazawa, Y., Iyomoto, N., Kubota, A., Matsumoto, Y., \& Makishima, K. 2001, A\&A, 374, 73 
Gallo, L. C., Brandt, W. N., Costantini, E., Fabian, A. C., Iwasawa, K., \& Papadakis, I. E. 2007, MNRAS, 377, 391

Gallo, L. C., Lehmann, I., Pietsch, W., Boller, T., Brinkmann, W., Friedrich, P., \& Grupe, D. 2006, MNRAS, 365, 688

Gondoin, P., Orr, A., Lumb, D., \& Santos-Lleo, M. 2002, A\&A, 388, 74

Goulding, A. D., \& Alexander, D. M. 2009, MNRAS, 398, 1165

Greenhill, L. J., Tilak, A., \& Madejski, G. 2008, ApJ, 686, L13

Guainazzi, M., Matt, G., \& Perola, G. C. 2005, A\&A, 444, 119

Iwasawa, K., Matt, G., Fabian, A. C., Bianchi, S., Brandt, W. N., Guainazzi, M., Murayama, T., \& Taniguchi, Y. 2001, MNRAS, 326, 119

Iyomoto, N., Fukazawa, Y., Nakai, N., \& Ishihara, Y. 2001, ApJ, 561, L69

Komossa, S., \& Fink, H. 1997, A\&A, 327, 555

Krolik, J. H., Madau, P., \& Zycki, P. T. 1994, ApJ, 420, L57

LaMassa, S. M., Heckman, T. M., Ptak, A., Hornschemeier, A., Martins, L., Sonnentrucker, P., \& Tremonti, C. 2009, ApJ, 705, 568

LaMassa, S. M., Heckman, T. M., Ptak, A., Martins, L., Wild, V., \& Sonnentrucker, P. 2010, ApJ, 720,786

Levenson, N. A., Heckman, T. M., Krolik, J. H., Weaver, K. A., \& Życki, P. T. 2006, ApJ, 648, 111

Lutz, D., Maiolino, R., Spoon, H. W. W., \& Moorwood, A. F. M. 2004, A\&A, 418, 465

Madejski, G., Done, C., Życki, P. T., \& Greenhill, L. 2006, ApJ, 636, 75

Maiolino, R., Salvati, M., Bassani, L., Dadina, M., della Ceca, R., Matt, G., Risaliti, G., \& Zamorani, G. 1998, A\&A, 338, 781

Malaguti, G., et al. 1998, A\&A, 331, 519

Matt, G., Bianchi, S., D’Ammando, F., \& Martocchia, A. 2004, A\&A, 421, 473

Matt, G., Guainazzi, M., Perola, G. C., Fiore, F., Nicastro, F., Cappi, M., \& Piro, L. 2001, A\&A, 377, L31

Meléndez, M., Kraemer, S. B., Schmitt, H. R., Crenshaw, D. M., Deo, R. P., Mushotzky, R. F., \& Bruhweiler, F. C. 2008a, ApJ, 689, 95

Meléndez, M., et al. 2008b, ApJ, 682, 94 
Miller, L., Turner, T. J., \& Reeves, J. N. 2009, MNRAS, 399, L69

Molendi, S., Bianchi, S., \& Matt, G. 2003, MNRAS, 343, L1

Murphy, K. D., \& Yaqoob, T. 2009, MNRAS, 397, 1549

Nandra, K. 2006, MNRAS, 368, L62

Nandra, K., \& George, I. M. 1994, MNRAS, 267, 974

Nandra, K., O’Neill, P. M., George, I. M., \& Reeves, J. N. 2007, MNRAS, 382, 194

Nenkova, M., Sirocky, M. M., Nikutta, R., Ivezić, Ž., \& Elitzur, M. 2008, ApJ, 685, 160

Netzer, H., Lemze, D., Kaspi, S., George, I. M., Turner, T. J., Lutz, D., Boller, T., \& Chelouche, D. 2005, ApJ, 629, 739

Noguchi, K., Terashima, Y., \& Awaki, H. 2009, ApJ, 705, 454

Pellegrini, S., Baldi, A., Fabbiano, G., \& Kim, D. 2003, ApJ, 597, 175

Pounds, K., \& Vaughan, S. 2006, MNRAS, 368, 707

Ptak, A., Heckman, T., Levenson, N. A., Weaver, K., \& Strickland, D. 2003, ApJ, 592, 782

Reeves, J. N., Nandra, K., George, I. M., Pounds, K. A., Turner, T. J., \& Yaqoob, T. 2004, ApJ, 602,648

Risaliti, G., et al. 2009, ApJ, 696, 160

Rothschild, R. E., et al. 2006, ApJ, 641, 801

Schurch, N. J., Roberts, T. P., \& Warwick, R. S. 2002, MNRAS, 335, 241

Schurch, N. J., Warwick, R. S., Griffiths, R. E., \& Sembay, S. 2003, MNRAS, 345, 423

Shinozaki, K., Miyaji, T., Ishisaki, Y., Ueda, Y., \& Ogasaka, Y. 2006, AJ, 131, 2843

Shu, X., Wang, J., \& Jiang, P. 2008, Chinese Journal of Astronomy and Astrophysics, 8, 204

Shu, X. W., Wang, J. X., Jiang, P., Fan, L. L., \& Wang, T. G. 2007, ApJ, 657, 167

Shu, X. W., Yaqoob, T., \& Wang, J. X. 2010, ApJS, 187, 581

Treister, E., Urry, C. M., \& Virani, S. 2009, ApJ, 696, 110

Turner, T. J., et al. 2002, ApJ, 574, L123

Vignati, P., et al. 1999, A\&A, 349, L57 
Watson, M. G., et al. 2009, A\&A, 493, 339

Wu, J., Vanden Berk, D. E., Brandt, W. N., Schneider, D., Gibson, R., \& Wu, J. 2009, ArXiv e-prints

Yaqoob, T., George, I. M., Kallman, T. R., Padmanabhan, U., Weaver, K. A., \& Turner, T. J. 2003, ApJ, 596, 85

Yaqoob, T., George, I. M., Nandra, K., Turner, T. J., Serlemitsos, P. J., \& Mushotzky, R. F. 2001, ApJ, 546, 759

Yaqoob, T., Murphy, K. D., Miller, L., \& Turner, T. J. 2010, MNRAS, 401, 411

Yaqoob, T., \& Padmanabhan, U. 2004, ApJ, 604, 63

Zhang, K., Wang, T., Dong, X., \& Lu, H. 2008, ApJ, 685, L109 
Table 1. Observations and Data

\begin{tabular}{|c|c|c|c|c|c|c|c|c|c|c|c|}
\hline Name & $\mathrm{z}$ & ObsID & Pileup;Radii & $\log L_{2-10 \mathrm{keV}}$ & $\log L_{[O I V]}$ & $\log L_{F e}$ & $E_{F e}(k e V)$ & $\sigma_{F e}(e V)$ & $\mathrm{EW}(\mathrm{eV})$ & Type & Ref \\
\hline 3C 120 & 0.033010 & 0109131101 & ; & 44.07 & 42.46 & $41.93_{-0.09}^{+0.08}$ & $6.42_{-0.02}^{+0.02}$ & $109.6_{-25.1}^{+22.6}$ & $67_{-12}^{+12}$ & $\mathrm{~S} 1$ & \\
\hline \multirow{22}{*}{$3 \mathrm{C} 273$} & \multirow{22}{*}{0.158339} & 0112770101 & 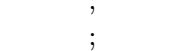 & \multirow{22}{*}{45.82} & \multirow{22}{*}{42.80} & \multirow{22}{*}{$<43.24$} & \multirow{22}{*}{$6.40^{*}$} & \multirow{22}{*}{$44^{*}$} & \multirow{22}{*}{$<17$} & \multirow{22}{*}{$\mathrm{S} 1$} & \\
\hline & & 0112770201 & $\mathrm{~m} 2$ & & & & & & & & \\
\hline & & 0112770501 & ; & & & & & & & & \\
\hline & & 0112770601 & ; & & & & & & & & \\
\hline & & 0112770701 & ; & & & & & & & & \\
\hline & & 0112770801 & ; & & & & & & & & \\
\hline & & 0112771001 & $\mathrm{~m} 2$ & & & & & & & & \\
\hline & & 0112771101 & $\mathrm{~m} 2$ & & & & & & & & \\
\hline & & 0126700101 & $\mathrm{~m} 1, \mathrm{~m} 2, \mathrm{pn} ;$ & & & & & & & & \\
\hline & & 0126700201 & $\mathrm{~m} 1, \mathrm{~m} 2, \mathrm{pn}$ & & & & & & & & \\
\hline & & 0126700301 & $\mathrm{~m} 1, \mathrm{~m} 2$ & & & & & & & & \\
\hline & & 0126700401 & $\mathrm{~m} 1, \mathrm{~m} 2, \mathrm{pn} ;$ & & & & & & & & \\
\hline & & 0126700501 & $\mathrm{~m} 1, \mathrm{~m} 2, \mathrm{pn}$ & & & & & & & & \\
\hline & & 0126700601 & $\mathrm{~m} 1, \mathrm{~m} 2$ & & & & & & & & \\
\hline & & 0126700701 & $\mathrm{~m} 1, \mathrm{~m} 2$ & & & & & & & & \\
\hline & & 0126700801 & $\mathrm{~m} 1, \mathrm{~m} 2$ & & & & & & & & \\
\hline & & 0136550101 & $\mathrm{~m} 1, \mathrm{~m} 2$ & & & & & & & & \\
\hline & & 0136550501 & $\mathrm{~m} 1, \mathrm{~m} 2$ & & & & & & & & \\
\hline & & 0136550801 & $\mathrm{~m} 1, \mathrm{~m} 2$ & & & & & & & & \\
\hline & & 0136551001 & $\mathrm{~m} 1, \mathrm{~m} 2$ & & & & & & & & \\
\hline & & 0159960101 & $\mathrm{~m} 1, \mathrm{~m} 2$ & & & & & & & & \\
\hline & & 0414190101 & $\mathrm{~m} 1, \mathrm{~m} 2$ & & & & & & & & \\
\hline \multirow[t]{2}{*}{ 3C 390.3} & \multirow[t]{2}{*}{0.056100} & 0203720201 & ; & \multirow[t]{2}{*}{44.47} & \multirow[t]{2}{*}{41.28} & \multirow[t]{2}{*}{$42.23_{-0.11}^{+0.10}$} & \multirow[t]{2}{*}{$6.43_{-0.02}^{+0.02}$} & \multirow[t]{2}{*}{$96.2_{-34.0}^{+40.7}$} & \multirow{2}{*}{$51_{-11}^{+13}$} & S1 & \\
\hline & & 0203720301 & ; & & & & & & & & \\
\hline Circinus & 0.001448 & 0111240101 & ; & 40.93 & 40.50 & $40.14_{-0.01}^{+0.01}$ & $6.39_{-0.00}^{+0.00}$ & $44.1_{-3.7}^{+2.5}$ & $1251_{-22}^{+22}$ & $\mathrm{CT}$ & 2 \\
\hline ESO 103-G035 & 0.013286 & 0109130601 & ; & 43.01 & 41.15 & $40.97_{-0.26}^{+0.17}$ & $6.47_{-0.03}^{+0.00}$ & $0.4_{-0.4}^{+53.9}$ & $51_{-23}^{+24}$ & $\mathrm{~S} 1$ & \\
\hline ESO 141-G055 & 0.036000 & 0101040501 & $\mathrm{~m} 1, \mathrm{~m} 2$ & 43.81 & 41.34 & $<41.75$ & $6.40^{*}$ & $44^{*-0.4}$ & $<73$ & $\mathrm{~S} 1$ & \\
\hline IC 2560 & 0.009757 & 0203890101 & ; & 40.99 & 41.06 & $40.32_{-0.04}^{+0.04}$ & $6.41_{-0.01}^{+0.00}$ & $<3.6$ & $1280_{-107}^{+114}$ & $\mathrm{CT}$ & 11 \\
\hline IC $4329 A$ & 0.016054 & 0101040401 & $\mathrm{~m} 1, \mathrm{~m} 2, \mathrm{pn}$ & 43.73 & 41.79 & $41.54_{-0.05}^{+0.02}$ & $6.41_{-0.01}^{+0.01}$ & $52.8_{-12.6}^{+9.4}$ & $52_{-5}^{+3}$ & $\mathrm{~S} 1$ & \\
\hline & & 0147440101 & $\mathrm{~m} 1, \mathrm{~m} 2$ & & & & & & & & \\
\hline IRAS01475-0740 & 0.017666 & 0200431101 & ; & 41.77 & 40.70 & $<40.42$ & $6.40^{*}$ & $44^{*}$ & $<405$ & S2 & 25 \\
\hline IRAS15091-2107 & 0.044607 & 0300240201 & ; & 43.63 & 42.16 & $<42.07$ & $6.40^{*}$ & $44^{*}$ & $<263$ & S1 & \\
\hline I ZW 1 & 0.061142 & 0110890301 & ; & 43.75 & 41.58 & $<41.47$ & $6.40^{*}$ & $44^{*}$ & $<50$ & $\mathrm{~S} 1$ & \\
\hline
\end{tabular}


Table 1-Continued

\begin{tabular}{|c|c|c|c|c|c|c|c|c|c|c|c|}
\hline Name & $\mathrm{z}$ & ObsID & Pileup;Radii & $\log L_{2-10 \mathrm{keV}}$ & $\log L_{[O I V]}$ & $\log L_{F e}$ & $E_{F e}(k e V)$ & $\sigma_{F e}(e V)$ & $\mathrm{EW}(\mathrm{eV})$ & Type & Ref \\
\hline & & 0300470101 & ; & \multirow{4}{*}{39.03} & \multirow{4}{*}{36.45} & \multirow{4}{*}{$<36.79$} & \multirow{4}{*}{$6.40^{*}$} & \multirow{4}{*}{$44^{*}$} & \multirow{4}{*}{$<110$} & \multirow{4}{*}{$\mathrm{S} 1$} & \\
\hline \multirow[t]{3}{*}{ M81 } & \multirow[t]{3}{*}{-0.00011} & 0112521001 & ; & & & & & & & & \\
\hline & & 0112521101 & ; & & & & & & & & \\
\hline & & 0200980101 & ;M1:30 & & & & & & & & \\
\hline MCG-2-58-22 & 0.046860 & 0109130701 & ; & 44.23 & 41.72 & $42.23_{-0.27}^{+0.20}$ & $6.41_{-0.08}^{+0.08}$ & $145.0_{-74.8}^{+136.3}$ & $90_{-41}^{+53}$ & $\mathrm{~S} 1$ & \\
\hline MCG-2-8-39 & 0.029894 & 0301150201 & ; & 42.28 & 41.49 & $41.21_{-0.14}^{+0.22}$ & $6.41_{-0.06}^{+0.08}$ & $76.5_{-75.9}^{+81.1}$ & $456_{-123}^{+303}$ & $\mathrm{~S} 2$ & 26 \\
\hline MCG-5-13-17 & 0.012445 & 0312190701 & ; & 42.17 & 40.64 & $40.83_{-0.12}^{+0.06}$ & $6.41_{-0.02}^{+0.02}$ & $21.2_{-23.0}^{+30.8}$ & $334_{-80}^{+49}$ & S1 & \\
\hline \multirow[t]{5}{*}{ MCG-6-30-15 } & \multirow[t]{5}{*}{0.007749} & 0111570101 & $\mathrm{~m} 2$ & \multirow[t]{5}{*}{42.74} & \multirow[t]{5}{*}{40.42} & \multirow{5}{*}{$40.68_{-0.13}^{+0.06}$} & \multirow{5}{*}{$6.44_{-0.01}^{+0.01}$} & \multirow{5}{*}{$141.5_{-10.0}^{+10.3}$} & \multirow{5}{*}{$82_{-20}^{+12}$} & \multirow[t]{5}{*}{$\mathrm{S} 1$} & \\
\hline & & 0111570201 & $\mathrm{~m} 2$ & & & & & & & & \\
\hline & & 0029740101 & $\mathrm{~m} 1$ & & & & & & & & \\
\hline & & 0029740701 & $\mathrm{~m} 1$ & & & & & & & & \\
\hline & & 0029740801 & $\mathrm{~m} 1$ & & & & & & & & \\
\hline \multirow[t]{9}{*}{ MRK 3} & \multirow[t]{9}{*}{0.013509} & 0111220201 & ; & \multirow[t]{9}{*}{42.39} & \multirow[t]{9}{*}{41.97} & \multirow{9}{*}{$41.22_{-0.03}^{+0.03}$} & \multirow{9}{*}{$6.42_{-0.01}^{+0.01}$} & \multirow{9}{*}{$38.7_{-10.7}^{+9.2}$} & $383_{-27}^{+31}$ & $\mathrm{CT}$ & 2 \\
\hline & & 0009220301 & ; & & & & & & & & \\
\hline & & 0009220401 & ; & & & & & & & & 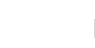 \\
\hline & & 0009220501 & ; & & & & & & & & 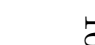 \\
\hline & & 0009220601 & ; & & & & & & & & 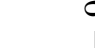 \\
\hline & & 0009220701 & ; & & & & & & & & \\
\hline & & 0009220901 & ; & & & & & & & & \\
\hline & & 0009221001 & ; & & & & & & & & \\
\hline & & 0009221601 & ; & & & & & & & & \\
\hline MRK 6 & 0.018813 & 0144230101 & ; & 43.09 & 41.61 & $41.04_{-0.12}^{+0.10}$ & $6.43_{-0.02}^{+0.02}$ & $0.6_{-0.6}^{+43.7}$ & $69_{-16}^{+17}$ & S1 & \\
\hline & & 0305600501 & ; & & & & & & & & \\
\hline MRK 79 & 0.022189 & 0103860801 & ; & 43.23 & 41.76 & $41.43_{-0.27}^{+0.20}$ & $6.39_{-0.04}^{+0.06}$ & $70.4_{-71.6}^{+86.6}$ & $142_{-65}^{+81}$ & S1 & \\
\hline & & 0103862101 & ; & & & & & & & & \\
\hline MRK 231 & 0.042170 & 0081340201 & ; & 42.49 & 41.62 & $41.24_{-0.25}^{+0.19}$ & $6.46_{-0.11}^{+0.11}$ & $187.3_{-85.5}^{+133.4}$ & $486_{-212}^{+264}$ & $\mathrm{CT}$ & 27 \\
\hline MRK 273 & 0.037780 & 0101640401 & ; & 42.15 & 42.32 & $41.34_{-0.29}^{+0.20}$ & $6.52_{-0.11}^{+0.09}$ & $261.6_{-123.8}^{+165.3}$ & $1249_{-615}^{+741}$ & $\mathrm{~S} 2$ & 28 \\
\hline MRK 335 & 0.025785 & 0101040101 & $\mathrm{~m} 1, \mathrm{~m} 2, \mathrm{pn}$ & 43.43 & 41.05 & $41.48_{-0.16}^{+0.12}$ & $6.44_{-0.03}^{+0.03}$ & $208.8_{-51.8}^{+123.8}$ & $113_{-34}^{+35}$ & S1 & \\
\hline & & 0306870101 & $\mathrm{~m} 1$ & & & & & & & & \\
\hline MRK 348 & 0.015034 & 0067540201 & ; & 43.19 & 41.09 & $41.23_{-0.22}^{+0.18}$ & $6.40_{-0.05}^{+0.03}$ & $57.1_{-56.5}^{+81.2}$ & $73_{-28}^{+38}$ & $\mathrm{~S} 2$ & 18 \\
\hline MRK 509 & 0.034397 & 0130720101 & $;$ & 44.00 & 41.89 & $41.94_{-0.11}^{+0.10}$ & $6.42_{-0.03}^{+0.03}$ & $124.2_{-40.6}^{+48.5}$ & $76_{-17}^{+19}$ & S1 & \\
\hline & & 0130720201 & m1;M1M2:30,PN:35 & & & & & & & & \\
\hline MRK 573 & 0.017179 & 0200430701 & $;$ & 41.45 & 41.72 & $40.36_{-0.15}^{+0.13}$ & $6.39_{-0.04}^{+0.02}$ & $<42.1$ & $754_{-216}^{+256}$ & $\mathrm{CT}$ & 12 \\
\hline MRK 609 & 0.034488 & 0103861001 & ; & 42.63 & 41.34 & $<41.28$ & $6.40^{*}$ & $44^{*}$ & $<398$ & $\mathrm{~S} 2$ & 19 \\
\hline MRK 841 & 0.036422 & 0112910201 & ; & 43.66 & 41.75 & $41.67_{-0.16}^{+0.13}$ & $6.42_{-0.04}^{+0.04}$ & $131.4_{-46.1}^{+58.8}$ & $97_{-29}^{+33}$ & S1 & \\
\hline
\end{tabular}


Table 1-Continued

\begin{tabular}{|c|c|c|c|c|c|c|c|c|c|c|c|c|}
\hline Name & $\mathrm{z}$ & ObsID & Pileup;Radii & $\log L_{2-10 k e V}$ & $\log L_{[O I V]}$ & $\log L_{F e}$ & $E_{F e}(k e V)$ & $\sigma_{F e}(e V)$ & $\mathrm{EW}(\mathrm{eV})$ & Type & Ref & \\
\hline & & 0205340401 & ; & & & & & & & & & \\
\hline & & 0070740101 & ; & & & & & & & & & \\
\hline & & 0070740301 & ; & & & & & & & & & \\
\hline NGC 424 & 0.011764 & 0002942301 & ; & 41.68 & 40.75 & $40.64_{-0.15}^{+0.13}$ & $6.39_{-0.02}^{+0.03}$ & $<38.7$ & $514_{-148}^{+178}$ & $\mathrm{CT}$ & 31 & \\
\hline NGC 513 & 0.019544 & 0301150401 & ; & 42.55 & 40.89 & $40.97_{-0.98}^{+0.156}$ & $6.33_{-0.08}^{+0.08}$ & $118.1_{-118.7}^{+90.4}$ & $183_{-163}^{+153}$ & $\mathrm{~S} 2$ & 26 & \\
\hline NGC 526A & 0.019097 & 0109130201 & ; & 43.28 & 41.20 & $41.11_{-0.21}^{+0.17}$ & $6.41_{-0.02}^{+0.03}$ & $75.1_{-75.5}^{+55.1}$ & $55_{-20}^{+26}$ & S1 & & \\
\hline & & 0150940101 & ; & & & & & & & & & \\
\hline NGC 777 & 0.016728 & 0203610301 & ; & 41.13 & $<40.44$ & $<40.02$ & $6.40^{*}$ & $44^{*}$ & $<1213$ & $\mathrm{~S} 2$ & 1 & \\
\hline & & 0304160301 & ; & & & & & & & & & \\
\hline NGC 985 & 0.043143 & 0150470601 & ; & 43.64 & 41.76 & $41.81_{-0.16}^{+0.13}$ & $6.44_{-0.03}^{+0.03}$ & $89.3_{-37.9}^{+37.6}$ & $128_{-40}^{+43}$ & S1 & & \\
\hline NGC 1068 & 0.003793 & 0111200101 & $\mathrm{~m} 1, \mathrm{pn}$ & 41.25 & 41.78 & $40.23_{-0.04}^{+0.03}$ & $6.41_{-0.01}^{+0.00}$ & $33.0_{-9.5}^{+7.8^{9}}$ & $532_{-44}^{+30}$ & $\mathrm{CT}$ & 2 & \\
\hline & & 0111200201 & $\mathrm{~m} 1, \mathrm{pn}$ & & & & & & & & & \\
\hline NGC 1194 & 0.013596 & 0307000701 & ; & 41.69 & 40.77 & $40.67_{-0.12}^{+0.11}$ & $6.40_{-0.02}^{+0.02}$ & $43.4_{-43.0}^{+38.3}$ & $468_{-114}^{+135}$ & $\mathrm{CT}$ & 29 & \\
\hline NGC 1275 & 0.017559 & 0305780101 & $\mathrm{~m} 1, \mathrm{~m} 2, \mathrm{pn}$ & 43.39 & $<41.11$ & $40.74_{-0.26}^{+0.19}$ & $6.40_{-0.02}^{+0.03}$ & $<60.1$ & $27_{-12}^{+15}$ & $\mathrm{~S} 2$ & 3 & \\
\hline & & 0085110101 & $\mathrm{~m} 1, \mathrm{~m} 2 ;$ & & & & & & & & & 1 \\
\hline NGC 1358 & 0.013436 & 0301650201 & ;PN:25 & 41.11 & 40.49 & $40.26_{-0.15}^{+0.14}$ & $6.44_{-0.03}^{+0.03}$ & $36.4_{-36.7}^{+50.4}$ & $956_{-278}^{+366}$ & $\mathrm{~S} 2$ & 5 & च \\
\hline NGC 1365 & 0.005457 & 0151370101 & ;PN:25 & 41.80 & 41.02 & $40.25_{-0.06}^{+0.06}$ & $6.38_{-0.01}^{+0.01}$ & $91.2_{-11.2}^{+0.0}$ & $168_{-21}^{+21}$ & S2 & 4 & I \\
\hline & & 0151370201 & ; & & & & & & & & & \\
\hline & & 0151370701 & ; & & & & & & & & & \\
\hline & & 0205590301 & ; & & & & & & & & & \\
\hline & & 0205590401 & ; & & & & & & & & & \\
\hline NGC 1386 & 0.002895 & 0140950201 & ; & 39.79 & 40.21 & $39.17_{-0.11}^{+0.10}$ & $6.39_{-0.02}^{+0.02}$ & $53.8_{-42.3}^{+29.7}$ & $1463_{-334}^{+383}$ & $\mathrm{CT}$ & 6 & \\
\hline NGC 2273 & 0.006138 & 0140951001 & ; & 40.92 & 40.09 & $40.37_{-0.23}^{+0.19}$ & $6.40_{-0.04}^{+0.04}$ & $<96.1$ & $1603_{-656}^{+865}$ & $\mathrm{CT}$ & 7 & \\
\hline NGC 2655 & 0.004670 & 0301650301 & ; & 40.80 & 39.48 & $<39.91$ & $6.40^{*}$ & $44^{*}$ & $<919^{-650}$ & $\mathrm{~S} 2$ & 8 & \\
\hline NGC 2992 & 0.007710 & 0147920301 & $\mathrm{~m} 1, \mathrm{~m} 2, \mathrm{pn}$ & 42.93 & 41.15 & $40.82_{-0.14}^{+0.11}$ & $6.40_{-0.02}^{+0.02}$ & $35.3_{-34.8}^{+31.1}$ & $51_{-13}^{+15}$ & $\mathrm{~S} 2$ & 9 & \\
\hline NGC 3079 & 0.003723 & 0110930201 & ; & 40.09 & 39.67 & $39.09_{-0.18}^{+0.17}$ & $6.50_{-0.05}^{+0.05}$ & $111.4_{-45.0}^{+58.5}$ & $695_{-238}^{+342}$ & $\mathrm{CT}$ & 10 & \\
\hline & & 0147760101 & ;PN:25 & & & & & & & & & \\
\hline NGC 3227 & 0.003859 & 0101040301 & ; & 41.49 & 40.27 & $39.91_{-0.07}^{+0.06}$ & $6.40_{-0.01}^{+0.01}$ & $43.0_{-43.0}^{+18.5}$ & $186_{-28}^{+28}$ & S1 & & \\
\hline NGC 3516 & 0.008836 & 0107460701 & ; & 42.46 & 40.99 & $40.51_{-0.13}^{+0.08}$ & $6.42_{-0.00}^{+0.00}$ & $<15.5$ & $66_{-17}^{+12^{\circ}}$ & S1 & & \\
\hline NGC 3783 & 0.009730 & 0112210101 & $\mathrm{~m} 1, \mathrm{~m} 2$ & 42.92 & 40.77 & $41.15_{-0.02}^{+0.02}$ & $6.40_{-0.00}^{+0.00}$ & $61.3_{-6.2}^{+5.7}$ & $108_{-5}^{+5}$ & $\mathrm{~S} 1$ & & \\
\hline & & 0112210201 & $\mathrm{~m} 2$ & & & & & & & & & \\
\hline & & 0112210501 & $\mathrm{~m} 2$ & & & & & & & & & \\
\hline NGC 3786 & 0.008933 & 0204650301 & ; & 42.12 & 40.52 & $<41.06$ & $6.40^{*}$ & $44^{*}$ & $<541$ & $\mathrm{~S} 2$ & 30 & \\
\hline NGC 3982 & 0.003699 & 0204651201 & ; & $<39.77$ & 39.55 & $<39.02$ & $6.40^{*}$ & $44^{*}$ & $<1184$ & $\mathrm{CT}$ & 12 & \\
\hline NGC 4051 & 0.002336 & 0157560101 & ; & 40.89 & 39.50 & $39.25_{-0.07}^{+0.07}$ & $6.41_{-0.01}^{+0.01}$ & $57.5_{-20.7}^{+18.9}$ & $177_{-26}^{+31}$ & $\mathrm{~S} 1$ & & \\
\hline
\end{tabular}


Table 1-Continued

\begin{tabular}{|c|c|c|c|c|c|c|c|c|c|c|c|}
\hline Name & $\mathrm{z}$ & ObsID & Pileup;Radii & $\log L_{2-10 k e V}$ & $\log L_{[O I V]}$ & $\log L_{F e}$ & $E_{F e}(k e V)$ & $\sigma_{F e}(e V)$ & $\mathrm{EW}(\mathrm{eV})$ & Type & Ref \\
\hline NGC 4138 & 0.002962 & 0112551201 & ; & 41.08 & 38.92 & $39.13_{-0.34}^{+0.21}$ & $6.38_{-0.04}^{+0.05}$ & $<87.4$ & $80_{-43}^{+48}$ & $\mathrm{~S} 2$ & 8 \\
\hline \multirow{7}{*}{ NGC 4151} & 0.003319 & 0112310101 & ; & 42.33 & 40.70 & $40.61_{-0.02}^{+0.02}$ & $\begin{array}{r}6.00_{-0.00}^{+0.00} \\
6.00\end{array}$ & $48.5_{-2.5}^{+4.4}$ & $117_{-4}^{+4}$ & S1 & \\
\hline & & 0112830201 & $\mathrm{~m} 1, \mathrm{~m} 2$ & & & & & & & & \\
\hline & & 0112830501 & ; & & & & & & & & \\
\hline & & 0143500101 & ; & & & & & & & & \\
\hline & & 0143500201 & $\mathrm{~m} 1, \mathrm{~m} 2$ & & & & & & & & \\
\hline & & 0143500301 & $\mathrm{~m} 1, \mathrm{~m} 2$ & & & & & & & & \\
\hline & & 0112190201 & ; & & & & & & & & \\
\hline NGC 4168 & 0.007388 & 0112550501 & ; & 40.12 & 39.23 & $<38.98$ & $6.40^{*}$ & $44^{*}$ & $<901$ & $\mathrm{~S} 2$ & 8 \\
\hline NGC 4235 & 0.008039 & 0204650201 & ; & 41.65 & 39.79 & $40.14_{-0.19}^{+0.15}$ & $6.41_{-0.03}^{+0.04}$ & $51.2_{-51.0}^{+49.7}$ & $284_{-98}^{+113}$ & S1 & \\
\hline \multirow[t]{6}{*}{ NGC 4258} & 0.001494 & 0110920101 & ; & 40.54 & 38.57 & $38.24_{-0.36}^{+0.19}$ & $6.40_{-0.06}^{+0.03}$ & $<98.8$ & $34_{-19}^{+20}$ & $\mathrm{~S} 2$ & 8 \\
\hline & & 0203270201 & ;PN:25 & & & & & & & & \\
\hline & & 0059140101 & ; & & & & & & & & \\
\hline & & 0059140201 & ; & & & & & & & & \\
\hline & & 0059140401 & ; & & & & & & & & \\
\hline & & 0059140901 & ; & & & & & & & & \\
\hline NGC 4378 & 0.008536 & 0301650801 & ; & $<40.54$ & 39.47 & $<39.45$ & $6.40^{*}$ & $44^{*}$ & $<1110$ & $\mathrm{~S} 2$ & 8 \\
\hline NGC 4388 & 0.008419 & 0110930701 & ; & 42.55 & 41.61 & $41.00_{-0.12}^{+0.11}$ & $6.43_{-0.02}^{+0.02}$ & $42.6_{-42.6}^{+27.6}$ & $156_{-38}^{+43}$ & $\mathrm{~S} 2$ & 13 \\
\hline \multirow[t]{4}{*}{ NGC 4395} & 0.001064 & 0112521901 & ; & 40.18 & 38.02 & $38.20_{-0.13}^{+0.11}$ & $6.37_{-0.03}^{+0.02}$ & $74.9_{-54.7}^{+38.7}$ & $87_{-23}^{+24}$ & $\mathrm{~S} 2$ & 8 \\
\hline & & 0112522701 & ; & & & & & & & & \\
\hline & & 0142830101 & ; & & & & & & & & \\
\hline & & 0200340101 & ; & & & & & & & & \\
\hline \multirow[t]{2}{*}{ NGC 4472} & 0.003326 & 0112550601 & ;PN:25 & 39.91 & $<39.21$ & $<38.17$ & $6.40 *$ & $44^{*}$ & $<229$ & $\mathrm{~S} 2$ & 8 \\
\hline & & 0200130101 & ; & & & & & & & & \\
\hline NGC 4477 & 0.004520 & 0112552101 & ; & 39.64 & 38.88 & $<38.81$ & $6.40^{*}$ & $44^{*}$ & $<1436$ & $\mathrm{~S} 2$ & 8 \\
\hline \multirow[t]{2}{*}{ NGC 4501} & 0.007609 & 0106060601 & ; & 40.13 & 39.71 & $<38.89$ & $6.40^{*}$ & $44^{*}$ & $<871$ & $\mathrm{CT}$ & 14 \\
\hline & & 0112550801 & ;M1M2PN:25 & & & & & & & & \\
\hline NGC 4507 & 0.011801 & 0006220201 & ; & 42.61 & 41.01 & $41.13_{-0.06}^{+0.05}$ & $6.39_{-0.01}^{+0.01}$ & $48.2_{-16.9}^{+12.2}$ & $176_{-21}^{+22}$ & $\mathrm{~S} 2$ & 15 \\
\hline NGC 4565 & 0.004103 & 0112550301 & ;M1M2PN:25 & 39.96 & 38.89 & $<38.65$ & $6.40^{*}$ & $44^{*-10.9}$ & $<386$ & $\mathrm{~S} 2$ & 8 \\
\hline NGC 4579 & 0.005067 & 0112840101 & ; & 41.39 & 39.21 & $39.57_{-0.26}^{+0.17}$ & $6.39_{-0.04}^{+0.04}$ & $0.7_{-0.7}^{+86.3}$ & $137_{-61}^{+66}$ & $\mathrm{~S} 2$ & 16 \\
\hline NGC 4593 & 0.009000 & 0109970101 & $\mathrm{~m} 2$ & 42.65 & 40.38 & $40.91_{-0.11}^{+0.26}$ & $\begin{array}{l}6.42_{-0.02}^{+0.04} \\
+0.01\end{array}$ & $0.1_{-0.2}^{+0.7}$ & $106_{-23}^{+61}$ & $\mathrm{~S} 1$ & \\
\hline NGC 4594 & 0.003416 & 0084030101 & ; & 40.63 & 38.83 & $<38.99$ & $6.40^{*}$ & $44^{*-2.2}$ & $<243$ & $\mathrm{~S} 2$ & 17 \\
\hline NGC 4639 & 0.003395 & 0112551001 & ; & 40.18 & 38.59 & $<39.08$ & $6.40^{*}$ & $44^{*}$ & $<871$ & $\mathrm{~S} 1$ & \\
\hline NGC 4698 & 0.003342 & 0112551101 & ; & 39.19 & 38.70 & $<38.09$ & $6.40^{*}$ & $44^{*}$ & $<834$ & $\mathrm{~S} 2$ & 8 \\
\hline NGC 4939 & 0.010374 & 0032141201 & ; & 42.02 & 41.01 & $<40.63$ & $6.40^{*}$ & $44^{*}$ & $<248$ & $\mathrm{~S} 2$ & 18 \\
\hline
\end{tabular}


Table 1-Continued

\begin{tabular}{|c|c|c|c|c|c|c|c|c|c|c|c|}
\hline Name & z & ObsID & Pileup;Radii & $\log L_{2-10 \mathrm{keV}}$ & $\log L_{[O I V]}$ & $\log L_{F e}$ & $E_{F e}(k e V)$ & $\sigma_{F e}(e V)$ & $\mathrm{EW}(\mathrm{eV})$ & Type & Ref \\
\hline NGC 4945 & 0.001878 & $\begin{array}{l}0112310301 \\
0204870101\end{array}$ & $\begin{array}{l}\text {;M1M2PN:25 } \\
\text {;M1M2PN:25 }\end{array}$ & 40.19 & 39.37 & $39.32_{-0.03}^{+0.04}$ & $6.40_{-0.00}^{+0.00}$ & $<4.4$ & $696_{-52}^{+59}$ & $\mathrm{CT}$ & 2 \\
\hline NGC 4968 & 0.009863 & $\begin{array}{l}0200660201 \\
0002940101\end{array}$ & ; & 40.86 & 40.81 & $40.12_{-0.14}^{+0.13}$ & $6.42_{-0.03}^{+0.02}$ & $<65.2$ & $1252_{-338}^{+430}$ & $\mathrm{CT}$ & 25 \\
\hline NGC 5005 & 0.003156 & 0110930501 & ; & 39.98 & 38.64 & $<38.63$ & $6.40^{*}$ & $44^{*}$ & $<431$ & $\mathrm{~S} 2$ & 19 \\
\hline NGC 5033 & 0.002919 & 0094360501 & ; & 40.97 & 39.48 & $39.31_{-0.18}^{+0.13}$ & $6.42_{-0.03}^{+0.02}$ & $<67.4$ & $206_{-68}^{+74}$ & $\mathrm{~S} 1$ & \\
\hline NGC 5128 & 0.001825 & $\begin{array}{l}0093650201 \\
0093650301\end{array}$ & $\begin{array}{l}\mathrm{m} 1, \mathrm{~m} 2, \mathrm{pn} ; \\
\mathrm{m} 2, \mathrm{pn} ;\end{array}$ & 41.94 & 39.86 & $39.85_{-0.07}^{+0.07}$ & $6.42_{-0.01}^{+0.01}$ & $30.7_{-30.7}^{+17.0}$ & $50_{-7}^{+\overline{8}^{\circ}}$ & $\mathrm{S} 2$ & 20 \\
\hline NGC 5194 & 0.001544 & 0112840201 & ; & 39.39 & 39.11 & $38.43_{-0.15}^{+0.12}$ & $6.43_{-0.02}^{+0.02}$ & $37.2_{-38.1}^{+37.3}$ & $703_{-202}^{+229}$ & $\mathrm{CT}$ & 21 \\
\hline NGC 5256 & 0.027863 & 0055990501 & ; & 41.81 & 42.04 & $40.57_{-0.22}^{+0.17}$ & $6.46_{-0.03}^{+0.03}$ & $<85.4$ & $418_{-165}^{+206}$ & $\mathrm{CT}$ & 25 \\
\hline NGC 5273 & 0.003549 & 0112551701 & ; & 41.35 & 39.01 & $39.68_{-0.27}^{+0.14}$ & $\begin{array}{l}6.43_{-0.03}^{+0.03} \\
\end{array}$ & $70.5_{-70.3}^{+50.0}$ & $212_{-97}^{+83}$ & $\mathrm{~S} 1$ & \\
\hline NGC 5506 & 0.006181 & \begin{tabular}{l|}
0013140101 \\
0013140201 \\
0201830201 \\
0201830301 \\
0201830401 \\
0201830501
\end{tabular} & $\begin{array}{c}; \\
\mathrm{m} 1, \mathrm{~m} 2 \\
; \\
; \\
; \\
;\end{array}$ & 42.80 & 41.27 & $40.56_{-0.08}^{+0.08}$ & $6.40_{-0.01}^{+0.01}$ & $20.4_{-20.4}^{+19.6}$ & $40_{-6}^{+\overline{7}^{9 i}}$ & $\mathrm{~S} 2$ & 22 \\
\hline NGC 5548 & 0.017175 & $\begin{array}{l}0109960101 \\
0089960301 \\
0089960401\end{array}$ & $\begin{array}{c}\mathrm{m} 2 \\
; \\
;\end{array}$ & 43.45 & 41.00 & $41.32_{-0.05}^{+0.06}$ & $6.41_{-0.01}^{+0.01}$ & $68.4_{-18.5}^{+17.4}$ & $66_{-7}^{+9}$ & S1 & \\
\hline NGC 5643 & 0.003999 & 0140950101 & ;M1M2PN:25 & 40.48 & 40.46 & $39.70_{-0.10}^{+0.09}$ & $6.41_{-0.02}^{+0.01}$ & $<48.9$ & $1139_{-240}^{+275}$ & $\mathrm{CT}$ & 6 \\
\hline NGC 6240 & 0.024480 & $\begin{array}{l}0101640101 \\
0101640601 \\
0147420201 \\
0147420401 \\
0147420501 \\
0147420601\end{array}$ & $\begin{array}{l}; \\
; \\
; \\
; \\
; \\
;\end{array}$ & 42.51 & 41.82 & $41.26_{-0.07}^{+0.07}$ & $6.41_{-0.01}^{+0.02}$ & $<40.6$ & $346_{-53}^{+57}$ & $\mathrm{CT}$ & 32 \\
\hline NGC 7172 & 0.008683 & $\begin{array}{l}0147920601 \\
0202860101\end{array}$ & $;$ & 42.58 & 40.91 & $40.68_{-0.09}^{+0.06}$ & $6.42_{-0.01}^{+0.01}$ & $65.4_{-20.4}^{+19.2}$ & $88_{-16}^{+13}$ & S2 & 18 \\
\hline NGC 7213 & 0.005839 & 0111810101 & $\mathrm{~m} 2$ & 42.20 & 39.20 & $40.13_{-0.07}^{+0.07}$ & $6.42_{-0.01}^{+0.01}$ & $8.3_{-8.3}^{+36.8}$ & $74_{-10}^{+13}$ & S1 & \\
\hline NGC 7314 & 0.004763 & 0111790101 & $\mathrm{~m} 1, \mathrm{~m} 2$ & 42.23 & 40.39 & $<40.17$ & $6.40^{*}$ & $44^{*}$ & $<64$ & $\mathrm{~S} 2$ & 23 \\
\hline NGC 7469 & 0.016317 & $\begin{array}{l}0112170101 \\
0112170301\end{array}$ & $\begin{array}{l}\mathrm{m} 1, \mathrm{~m} 2 \\
\mathrm{~m} 1, \mathrm{~m} 2\end{array}$ & 43.13 & 41.34 & $41.24_{-0.09}^{+0.07}$ & $6.41_{-0.02}^{+0.02}$ & $64.1_{-29.1}^{+21.9}$ & $99_{-18}^{+18}$ & S1 & \\
\hline $\begin{array}{l}\text { NGC } 7479 \\
\text { NGC } 7582\end{array}$ & $\begin{array}{l}0.007942 \\
0.005254\end{array}$ & $\begin{array}{l}0025541001 \\
0112310201\end{array}$ & ; & $\begin{array}{r}<40.55 \\
41.27\end{array}$ & $\begin{array}{l}40.57 \\
41.13\end{array}$ & $\begin{array}{l}39.81_{-0.46}^{+0.20} \\
40.05_{-0.05}^{+0.04}\end{array}$ & $\begin{array}{l}6.45_{-0.04}^{+0.04} \\
6.41_{-0.01}^{+0.01}\end{array}$ & $\begin{array}{l}78.6_{-78.6}^{+63.2} \\
29.1_{-29.1}^{+13.4}\end{array}$ & $\begin{array}{l}1016_{-666}^{+604} \\
343_{-33}^{+31}\end{array}$ & $\begin{array}{r}\mathrm{S} 2 \\
\mathrm{CT}\end{array}$ & $\begin{array}{l}8 \\
2\end{array}$ \\
\hline
\end{tabular}


Table 1 - Continued

\begin{tabular}{|c|c|c|c|c|c|c|c|c|c|c|c|}
\hline Name & $\mathrm{z}$ & ObsID & Pileup;Radii & $\log L_{2-10 k e V}$ & $\log L_{[O I V]}$ & $\log L_{F e}$ & $E_{F e}(k e V)$ & $\sigma_{F e}(e V)$ & $\mathrm{EW}(\mathrm{eV})$ & Type & Ref \\
\hline & & 0204610101 & ; & & & & & & & & \\
\hline \multirow[t]{2}{*}{ NGC 7590} & 0.005255 & 0112310201 & ; & 40.12 & 39.62 & $<38.98$ & $6.40^{*}$ & $44^{*}$ & $<632$ & $\mathrm{CT}$ & 24 \\
\hline & & 0204610101 & ; & & & & & & & & \\
\hline \multirow[t]{2}{*}{ NGC 7603} & 0.029524 & 0066950301 & ; & 43.59 & 41.15 & $<42.06$ & $6.40^{*}$ & $44^{*}$ & $<302$ & S1 & \\
\hline & & 0066950401 & ; & & & & & & & & \\
\hline NGC 7674 & 0.028924 & 0200660101 & $;$ & 42.20 & 41.95 & $<41.20$ & $6.40^{*}$ & $44^{*}$ & $<531$ & $\mathrm{CT}$ & 33 \\
\hline UGC 12138 & 0.024974 & 0103860301 & ;M1:25,M2:35 & 43.13 & 41.20 & $<41.78$ & $6.40^{*}$ & $44^{*}$ & $<515$ & $\mathrm{~S} 2$ & 19 \\
\hline
\end{tabular}

Note. - Column(1): Name of object. Column(2): redshift. Column(3): XMM-Newton observation ID. Column(4): before the semicolon: which of the 3 EPIC detectors' exposures are suffering significant pileup. After the semicolon: in which of the 3 EPIC detectors' exposures, source region radius are reduced, instead of being the default 40 arcsec. M1 and M2 are abbreviations for MOS1 and MOS2. The number after the colon represents the extraction region radius in arcsec. Column(5-6): 2-10 keV and [OIV] luminosities in erg/s. Column(7-10): luminosity, central energy, $\sigma$ and equivalent width of the narrow Fe K $\alpha$ line in rest-frame with $90 \%$ errors. The upper limits to $\sigma$ are at $90 \%$ confidence level. $3 \sigma$ upper limits of luminosity and equivalent width are given when the line is not detected. * Denotes fixed quantities. Column(11): Seyfert type. S1: Seyfert 1; S2: Compton-Thin Seyfert 2; CT: Compton-Thick Sy2. Column(12): References for the X-ray classification of Sy2 galaxies (Compton-thin or Compton-thick).

References. - (1) this work; (2) Treister et al. 2009; (3) Churazov et al. 2003; (4) Risaliti et al. 2009; (5) Cardamone et al. 2007; (6) Maiolino et al. 1998; (7) Awaki et al. 2008; (8) Akvlas \& Georgantopoulos 2009; (9) Brenneman \& Revnolds 2009; (10)Ivomoto et al. 2001; (11) Madeiski et al. 2006; (12)Shu et al. 2007; (13)Beckmann et al. 2004; (14)Brightman \& Nandra 2008; (15) Matt et al. 2004; (16)Dewangan et al. 2004; (17)Pellegrini et al. 2003; (18) Noguchi et al. 2009; (19) Gallo et al. 2006; (20) Rothschild et al. 2006; (21) Fukazawa et al. 2001; (22) Shinozaki et al. 2006; (23) Branduardi-Ravmont et al. 2002; (24)Bassani et al. 1999; (25) Guainazzi et al. 2005; (26) Shu et al. 2008; (27) Braito et al. 2004; (28) Balestra et al. 2005; (29) Greenhill et al. 2008; (30)Komossa \& Fink 1997; (31)Iwasawa et al. 2001; (32)Vignati et al. 1999; (33) Malaguti et al. 1998; 
Table 2. Additional Gauss lines

\begin{tabular}{|c|c|c|c|c|}
\hline Name & $\begin{array}{c}\text { Energy } \\
(\mathrm{keV})\end{array}$ & $\begin{array}{c}\sigma \\
(e V)\end{array}$ & $\begin{array}{c}\text { Flux } \\
\left(10^{-6} \text { photons } / \mathrm{cm}^{-2} / \mathrm{s}^{-1}\right)\end{array}$ & References \\
\hline $3 \mathrm{C} 120$ & $6.96_{-0.03}^{+0.03}$ & $<72$ & $9_{-3}^{+5}$ & Ballantyne et al. (2004) \\
\hline Circinus & $\begin{array}{l}7.04_{-0.01}^{+0.01} \\
7.47_{-0.01}^{+0.01} \\
6.69_{-0.01}^{+0.01}\end{array}$ & $\begin{array}{c}39_{-7}^{+12} \\
<31 \\
67_{-14}^{+14}\end{array}$ & $\begin{array}{l}41_{-2}^{+2} \\
11_{-1}^{+2} \\
26_{-3}^{+3}\end{array}$ & Molendi et al. (2003) \\
\hline I ZW 1 & $6.83_{-0.10}^{+0.11}$ & $312_{-115}^{+128}$ & $8_{-1}^{+3}$ & Gallo et al. (2007) \\
\hline MCG-6-30-15 & $\begin{array}{l}5.97_{-0.05}^{+0.09} \\
6.89_{-0.02}^{+0.01}\end{array}$ & $\begin{array}{c}310_{-102}^{+98} \\
<27\end{array}$ & $\begin{array}{c}24_{-5}^{+11} \\
6_{-1}^{+1}\end{array}$ & Fabian \& Vaughan (2003) \\
\hline MRK 335 & $7.02_{-0.04}^{+0.03}$ & $<58$ & $5_{-2}^{+\frac{1}{3}}$ & Gondoin et al. (2002) \\
\hline NGC 1068 & $\begin{array}{l}6.69_{-0.01}^{+0.04} \\
7.01_{-0.02}^{+0.02}\end{array}$ & $\begin{array}{l}63_{-14}^{+14} \\
68_{-23}^{+18}\end{array}$ & $\begin{array}{l}33_{-3}^{+2} \\
15_{-3}^{+3}\end{array}$ & Pounds \& Vaughan (2006) \\
\hline NGC 1275 & $\begin{array}{l}6.69_{-0.01}^{+0.01} \\
6.98_{-0.03}^{+0.03}\end{array}$ & $\begin{array}{l}<16 \\
<58\end{array}$ & $\begin{array}{c}51_{-5}^{+3} \\
5_{-3}^{+3}\end{array}$ & Churazov et al. (2003) \\
\hline NGC 1365 & $5.78_{-0.06}^{+0.09}$ & $435_{-86}^{+55}$ & $67_{-24}^{+16}$ & Risaliti et al. (2009) \\
\hline NGC 3516 & $6.42_{-0.01}^{+0.02}$ & $127_{-23}^{+14}$ & $36_{-8}^{+3}$ & Turner et al. (2002) \\
\hline NGC 3783 & $7.03_{-0.02}^{+0.02}$ & $67_{-25}^{+22}$ & $16_{-3}^{+3}$ & Reeves et al. (2004) \\
\hline NGC 4151 & $7.08_{-0.03}^{+0.02}$ & $<65$ & $5_{-3}^{+4}$ & Schurch et al. (2003) \\
\hline NGC 4945 & $6.68_{-0.03}^{+0.03}$ & $218_{-19}^{+26}$ & $18_{-2}^{+3}$ & Schurch et al. (2002) \\
\hline NGC 5506 & $\begin{array}{l}6.53_{-0.06}^{+0.05} \\
6.99_{-0.02}^{+0.03}\end{array}$ & $\begin{array}{c}273_{-40}^{+37} \\
<73\end{array}$ & $\begin{array}{c}85_{-18}^{+19} \\
11_{-3}^{+6}\end{array}$ & Matt et al. (2001) \\
\hline NGC 6240 & $6.67_{-0.02}^{+0.02}$ & $<54$ & $7_{-1}^{+2}$ & Netzer et al. (2005) \\
\hline NGC 7314 & $6.68_{-0.12}^{+0.21}$ & $360_{-111}^{+97}$ & $45_{-16}^{+\frac{1}{2} 6}$ & Yaqoob et al. (2003) \\
\hline
\end{tabular}

Note. - Column(1): Name. Column(2-4): Centroid energy, $\sigma$ and flux of the line. The errors and upper limits are in 90\% confidence level. Column(5): References in which the detections of the additional lines were also reported. 

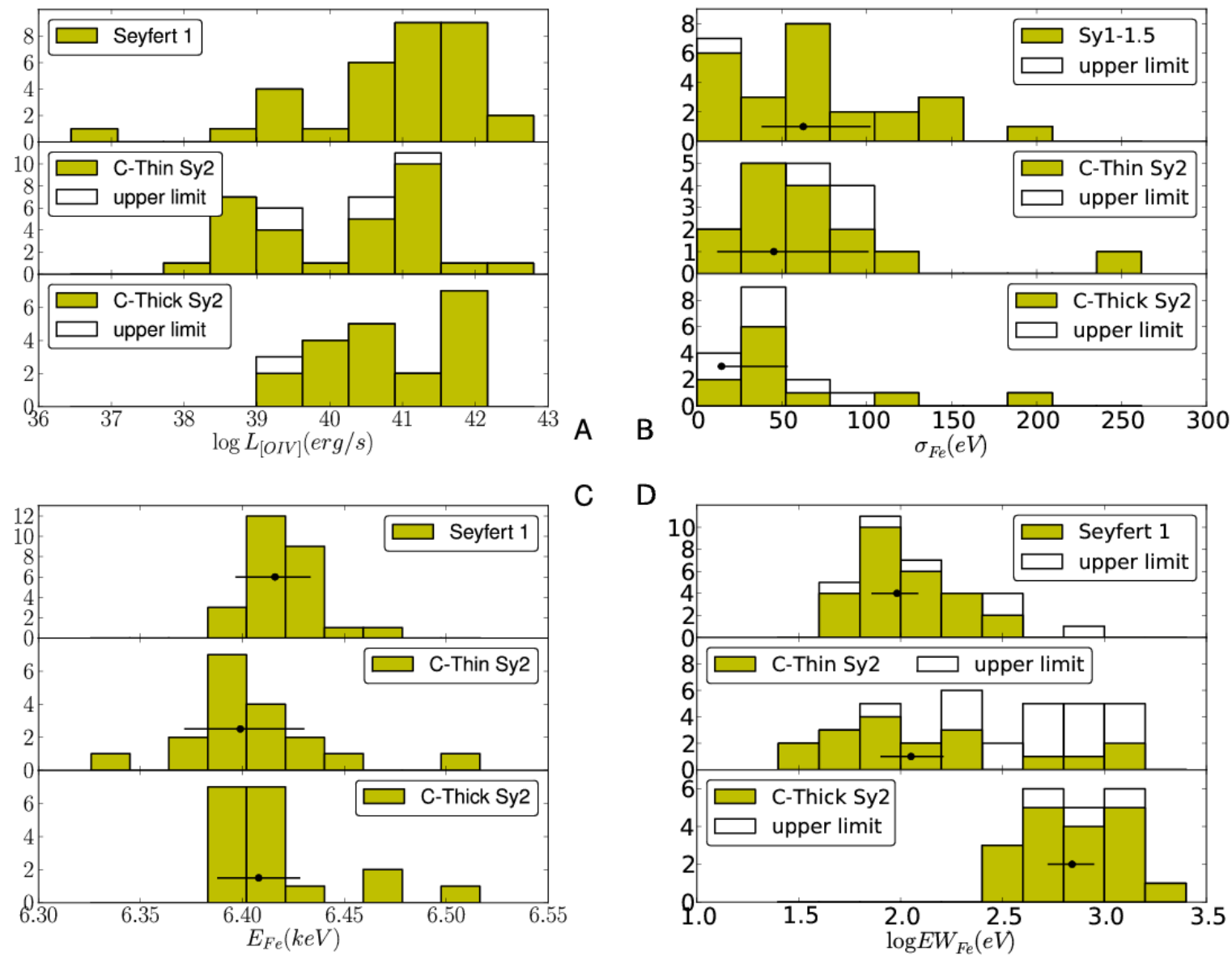

C D

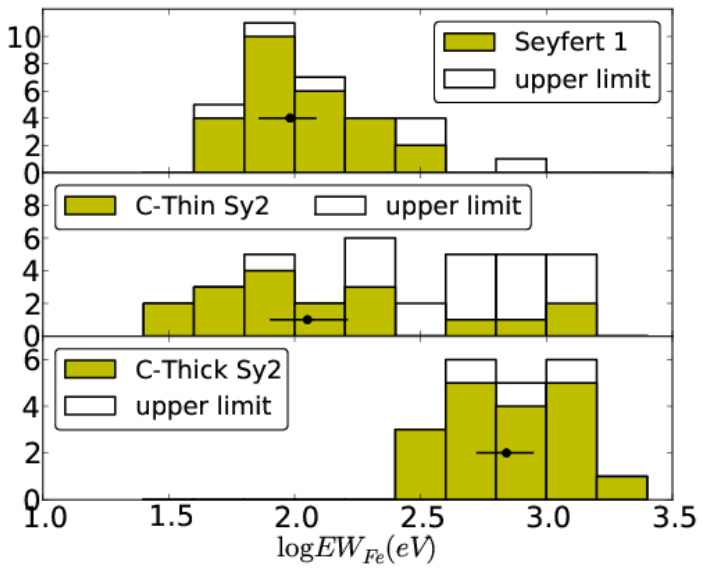

Fig. 1. - The histograms of [OIV] line luminosities and centroid narrow Fe K $\alpha$ line energies, $\sigma$ and EW. In panel B, C and D, we plot the median values and errorbars of the sources with narrow Fe $\mathrm{K} \alpha$ line detected. 


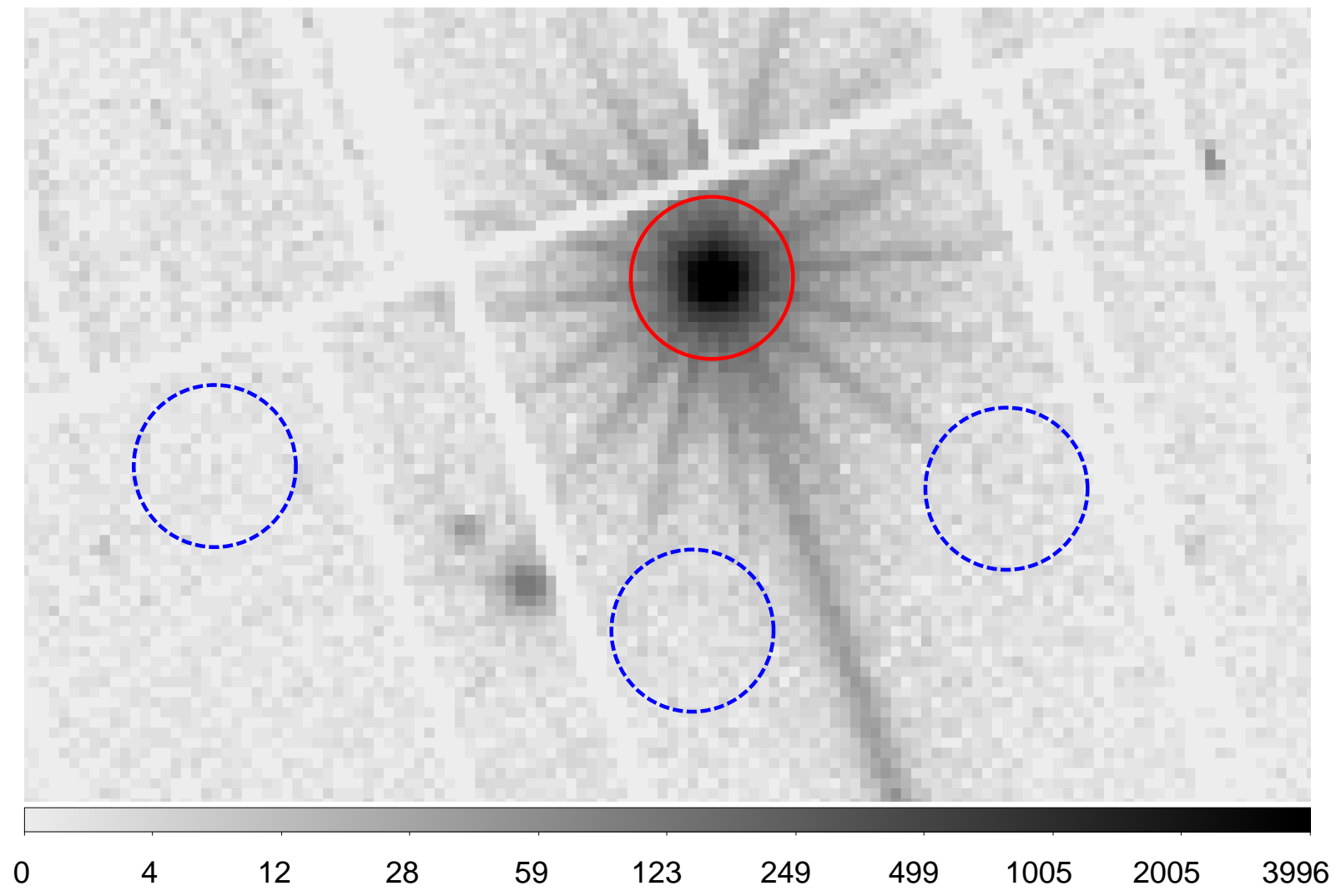

Fig. 2.- Example of source and background regions used to extract spectra (NGC 2992, ObsID 0147920301, PN detector), with single-pixel events only. The colorbar refers to the photon counts in each pixel. 

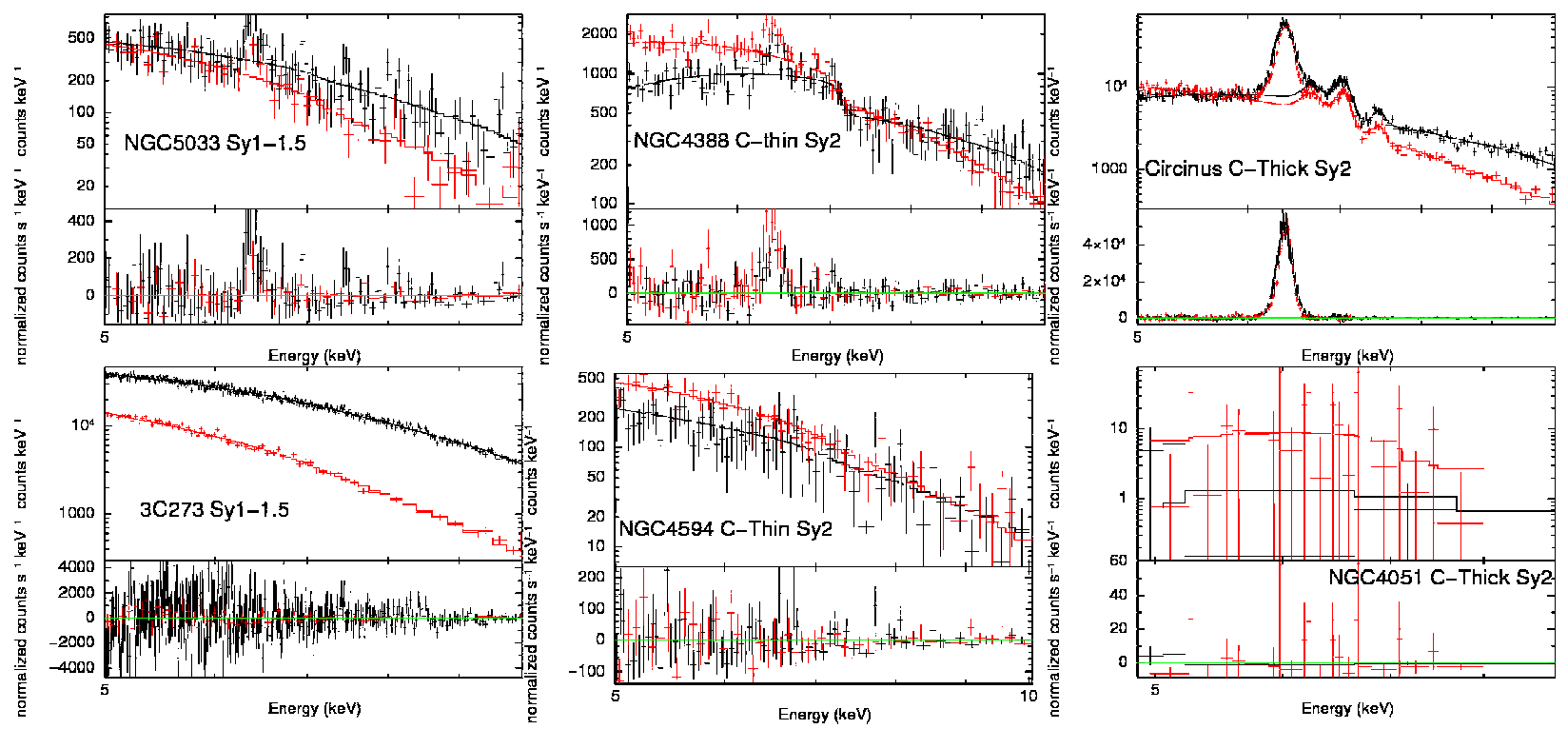

Fig. 3.- Sample spectra of our sample, including two Seyfert 1-1.5 galaxies, two Compton-thin Seyfert 2 galaxies, and two Compton-thick Seyfert 2 galaxies. The black and red data points refer to PN and MOS spectra respectively. All the model components but the narrow Fe $\mathrm{K} \alpha$ line are over plotted to show the Fe $\mathrm{K} \alpha$ line in the data to model ratio plot. The upper three sources have Fe K $\alpha$ line detected, and the lower three do not. 


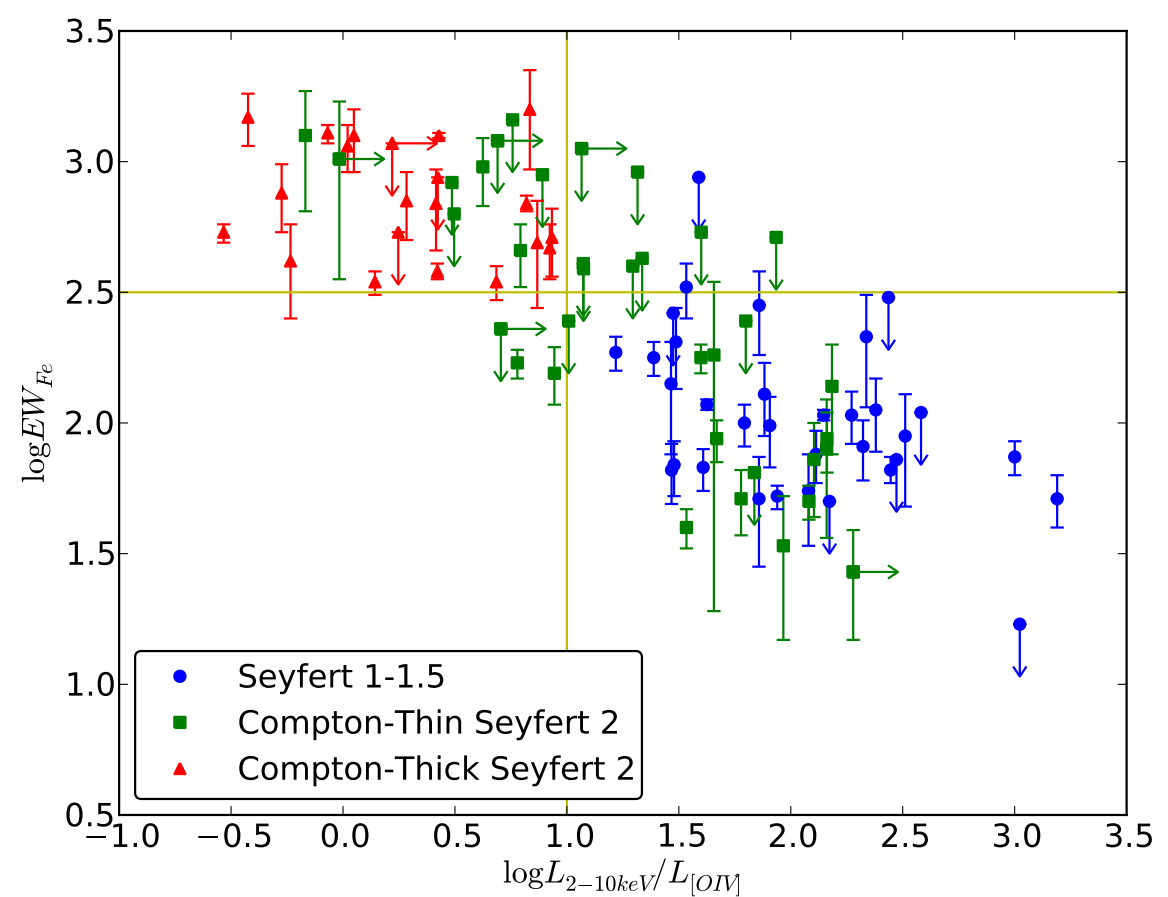

Fig. 4.- Narrow Fe K $\alpha$ line equivalent width versus $L_{2-10 k e V} / L_{[O I V]}$. The yellow lines can be used as plausible boundaries for Compton-Thick sources. 


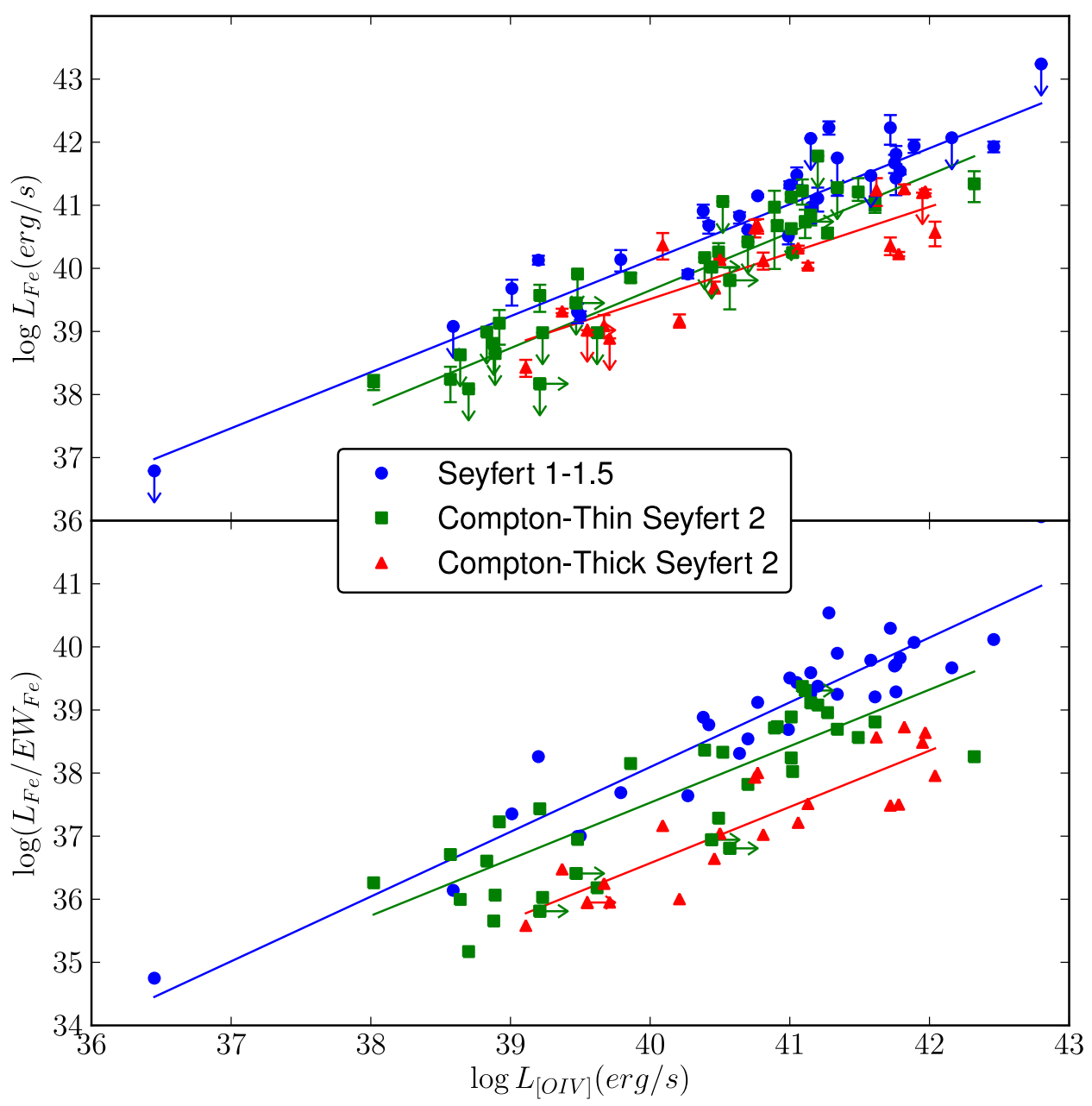

Fig. 5.- Upper panel: Narrow Fe K $\alpha$ line luminosity versus $L_{[O I V]}$. For the sources with narrow $\mathrm{Fe} \mathrm{K} \alpha$ lines detected, $90 \%$ errors of the luminosities are plotted. When the narrow Fe $\mathrm{K} \alpha$ line is not detected, $3 \sigma$ upper limit of the luminosity is given. Lower panel: $6.4 \mathrm{keV}$ monochromatic luminosity versus $L_{[O I V]}$. In both panels, the best-fit lines for Sy1s, Compton-Thin Sy2s and Compton-Thick Sy2s are shown as blue, green and red lines. 


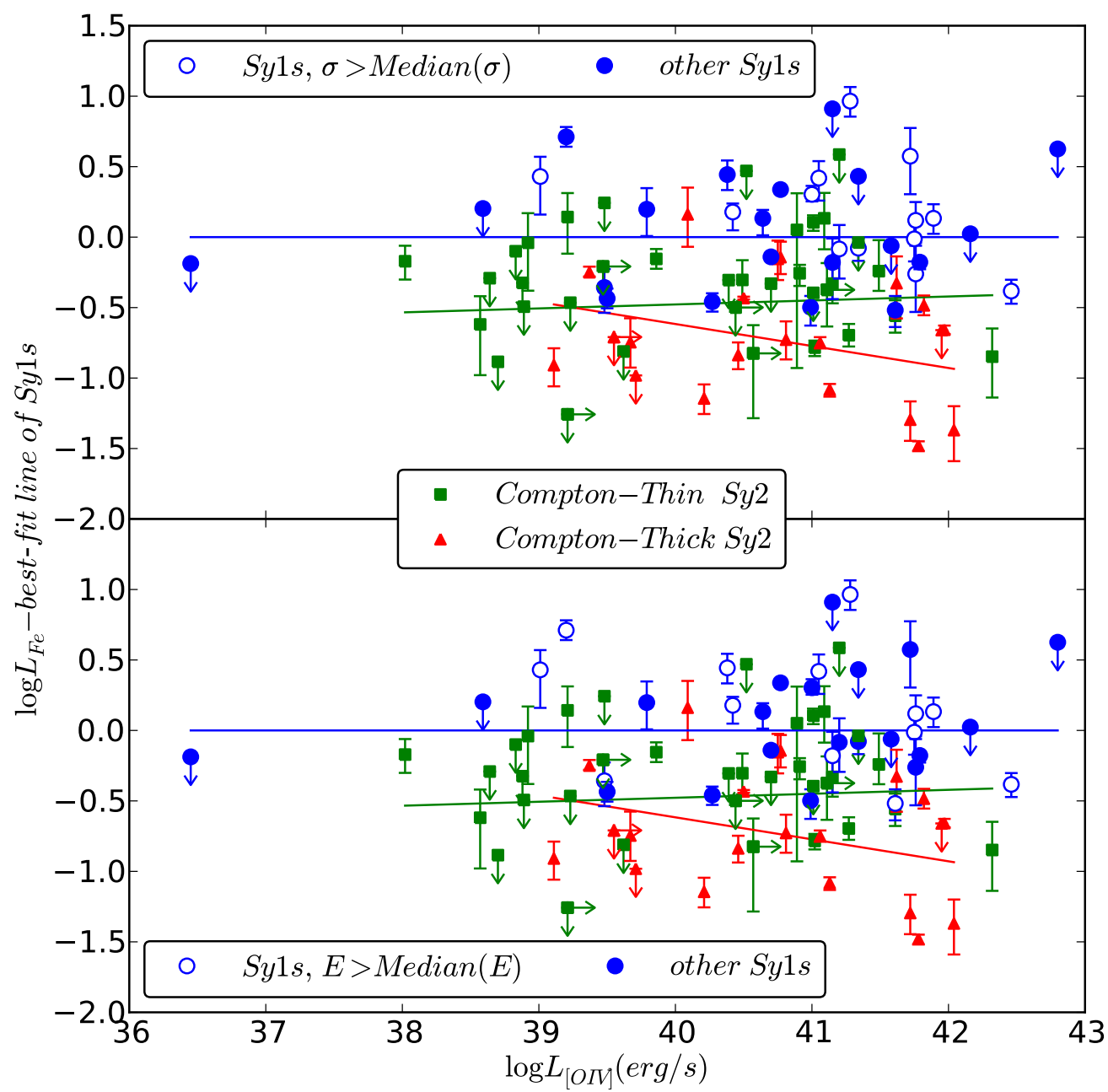

Fig. 6.- The deviation of $\log L_{F e}$ from the best-fit $\log L_{F e}-\log L_{O[I V]}$ line of Seyfert 1 galaxies, where we clearly see weaker narrow Fe K $\alpha$ emission line in Seyfert 2 galaxies. Best-fit $\log L_{F e}-$ $\log L_{O[I V]}$ correlations for Compton-thin and Compton-thick Sy2s are over-plotted. We also divide Sy1s by the Fe K $\alpha$ line width and centroid energy using the median value of the Sy1s with Fe K $\alpha$ line detected as a boundary, which demonstrate that Sy1s with larger Fe K $\alpha$ line width or centroid energy do not show obviously stronger line emission. 


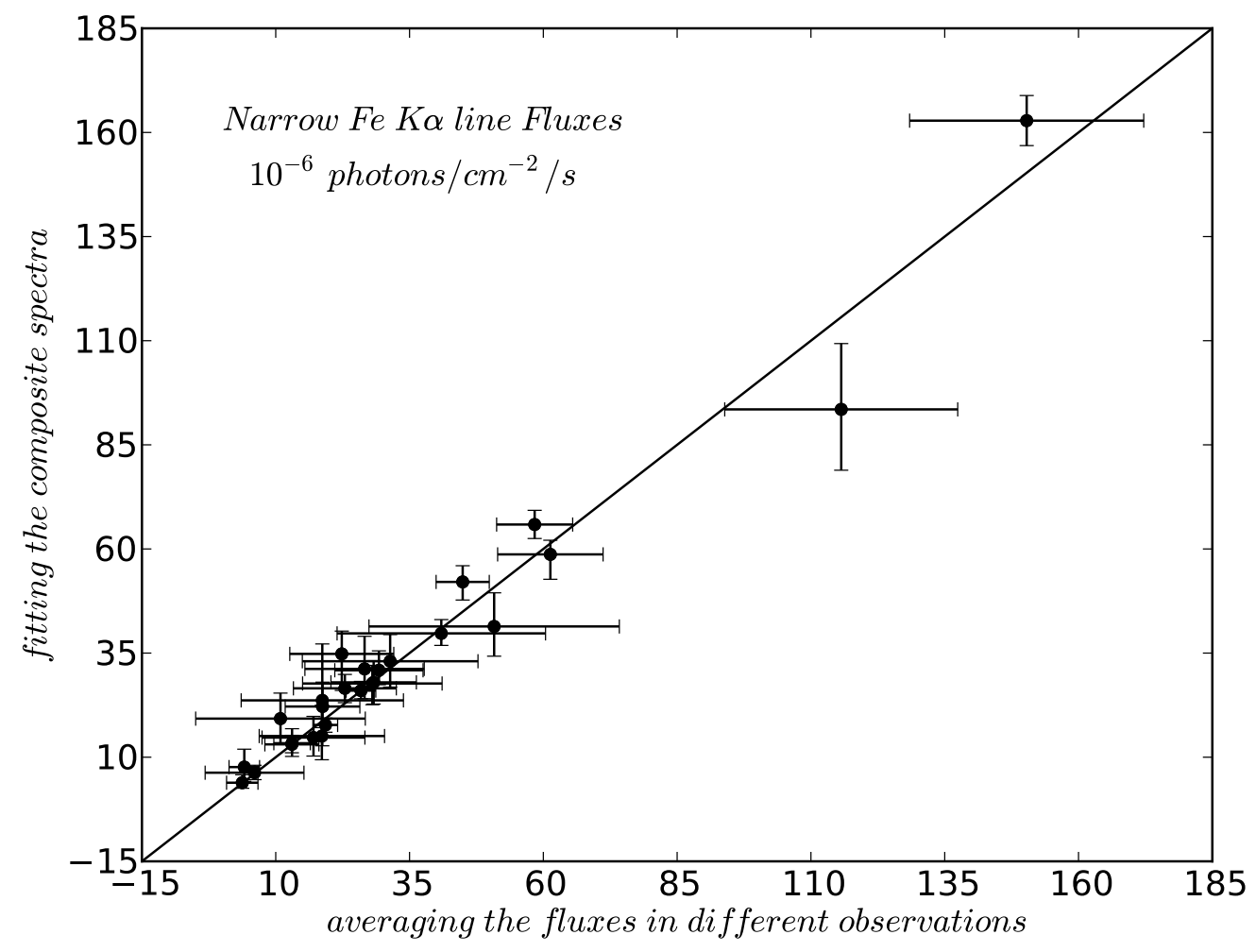

Fig. 7.- For sources with multiple XMM observations, spectral fitting to composite spectra yield narrow Fe $\mathrm{K} \alpha$ line flux consistent with the weighted mean of individual exposures. The line represents a slope of unity. 